\title{
Monotone Comparative Statics Under Uncertainty
}

\section{Citation}

Athey, Susan. 2002. Monotone comparative statics under uncertainty. Quarterly Journal of Economics 117(1): 187-223.

\section{Published Version}

http://dx.doi.org/10.1162/003355302753399481

\section{Permanent link}

http://nrs.harvard.edu/urn-3:HUL.InstRepos:3372263

\section{Terms of Use}

This article was downloaded from Harvard University's DASH repository, and is made available under the terms and conditions applicable to Other Posted Material, as set forth at http:// nrs.harvard.edu/urn-3:HUL.InstRepos:dash.current.terms-of-use\#LAA

\section{Share Your Story}

The Harvard community has made this article openly available.

Please share how this access benefits you. Submit a story.

Accessibility 


\title{
MONOTONE COMPARATIVE STATICS UNDER UNCERTAINTY*
}

\author{
Susan Athey
}

This paper analyzes monotone comparative statics predictions in several classes of stochastic optimization problems. The main results characterize necessary and sufficient conditions for comparative statics predictions to hold based on properties of primitive functions, that is, utility functions and probability distributions. The results apply when the primitives satisfy one of the following two properties: (i) a single-crossing property, which arises in applications such as portfolio investment problems and auctions, or (ii) log-supermodularity, which arises in the analysis of demand functions, affiliated random variables, stochastic orders, and orders over risk aversion.

\section{INTRODUCTION}

Since Samuelson, economists have studied and applied systematic tools for deriving comparative statics predictions. Recently, the theory of comparative statics has received renewed attention [Topkis 1978; Milgrom and Roberts 1990a, 1990b, 1994; Milgrom and Shannon 1994; Vives 1990]. The new literature provides general and widely applicable theorems about comparative statics, and further, it emphasizes the robustness of conclusions to changes in the specification of models. The literature shows that many of the robust comparative statics results that arise in economic theory rely on three main properties: supermodularity, log-supermodularity, and single-crossing properties. ${ }^{1}$

This paper characterizes log-supermodularity and singlecrossing properties in stochastic problems, establishing necessary

* I am indebted to Paul Milgrom and John Roberts for their advice and encouragement on my Ph.D. thesis, on which this paper is based. For helpful discussions, I would like to thank Kyle Bagwell, Peter Diamond, Joshua Gans, Edward Glaeser, Christian Gollier, Bengt Holmstrom, Ian Jewitt, Miles Kimball, Jonathan Levin, Eric Maskin, Preston McAfee, Edward Schlee, Christina Shannon, Scott Stern, several anonymous referees, and seminar participants at the Australian National University, Harvard/MIT Theory Workshop, Pennsylvania State University, the University of Montreal, Yale University, the 1997 summer meetings of the econometric society in Pasadena, and the 1997 summer meeting of the NBER Asset Pricing group. Generous financial support was provided by the National Science Foundation (graduate fellowship and Grant SBR9631760), the State Farm Companies Foundation, and the Massachusetts Institute of Technology.

1. A function on a product set is supermodular if increasing any one variable increases the returns to increasing each of the other variables; for differentiable functions this corresponds to nonnegative cross-partial derivatives between each pair of variables. A positive function is log-supermodular if the log of that function is supermodular.

( 2002 by the President and Fellows of Harvard College and the Massachusetts Institute of Technology.

The Quarterly Journal of Economics, February 2002 
and sufficient conditions for comparative statics predictions. ${ }^{2}$ More precisely, let $u$ be an agent's payoff function, let $f$ be a probability density function, and let the agent's objective function be given by $U(\mathbf{x}, \theta)=\int u(\mathbf{x}, \mathbf{s}) f(\mathrm{~s}, \theta) d \mathrm{~s}$, where $\mathbf{x}$ represents a choice vector and $\theta$ represents an exogenous parameter. The comparative statics question concerns conditions on primitives-the payoff function and probability density- under which the agent's optimal choice of $\mathbf{x}$ is nondecreasing in $\theta$.

We begin with families of problems where one of the primitive functions is log-supermodular (abbreviated log-spm). For example, an agent's marginal utility $u^{\prime}(w+s)$ is log-spm in $(w, s)$ if the utility function satisfies decreasing absolute risk aversion; a parameterized demand function $D(p ; \epsilon)$ is log-spm if demand becomes less elastic as $\epsilon$ increases; a set of random variables is affiliated $^{3}$ if the joint density is log-spm; and a parameterized density of a single random variable, $f(s ; \theta)$, is log-spm if the parameter shifts the distribution according to the monotone likelihood ratio property.

Our first result establishes that the agent's choice of $\mathbf{x}$ is nondecreasing in $\theta$ for all utility functions $u$ that are log-spm, if and only if $f$ is log-spm. One application considers a pricing game between firms with private information about their marginal costs; we provide conditions under which each firm's price increases in its marginal cost (and thus, a pure strategy Nash equilibrium exists). More generally, we show that $U$ is log-spm for all $u$ that are log-spm, if and only if $f$ is log-spm. The characterizations of log-spm are used to establish relationships between several commonly used orders over distributions in investment theory, as well as to show that decreasing absolute risk aversion is preserved in the presence of independent or affiliated background risks.

Our second set of comparative statics theorems provides necessary and sufficient conditions for comparative statics predictions in problems with a single random variable, where one of the

2. Vives [1990] and Athey [1998] study supermodularity in stochastic problems. Vives [1990] shows that supermodularity is preserved by integration. Athey [1998] allows for payoff functions that satisfy properties which are preserved by convex combinations (including supermodularity, monotonicity, and concavity). Independently, Gollier and Kimball [1995a, 1995b] use convex analysis to analyze comparative statics of investment problems. The properties studied in this paper are not preserved by convex combinations, and so different techniques are used.

3. A set of random variables is affiliated if, for all nondecreasing transformations, each pair is positively correlated. 
primitive functions satisfies a single-crossing property and the other is log-spm. ${ }^{4}$ The results are applied to portfolio and firm investment problems. ${ }^{5}$ We further show how the results change when we impose additional structure (such as restrictions on risk preferences).

Finally, we extend the analysis to problems of the form $\int v(x, y, s) f(s, \theta) d s$, where we characterize single crossing of the $x-y$ indifference curves; the results are applied to signaling games and consumption-savings problems.

\section{Comparative Statics with Log-Spm Primitives}

This section considers problems where one or both of the primitives, $u$ and $f$, are assumed to be nonnegative and log-spm.

\section{A. The Comparative Statics Problem}

We begin with some notation. Let $\Theta \subseteq \mathbb{R}$, let $X=X_{1} \times \cdots \times$ $X_{n}\left(\right.$ each $\left.X_{i} \subseteq \mathbb{R}\right){ }^{6}$ and let $S=S_{1} \times \cdots \times S_{m}$ (each $\left.S_{i} \subseteq \mathbb{R}\right)$. Let $\mu$ be a nonnegative $\sigma$-finite product measure on $S$, and let $u: X \times$ $S \rightarrow \mathbb{R}$ and $f: S \times \Theta \rightarrow \mathbb{R}$ be bounded, measurable functions. ${ }^{7}$ Define $U: X \times \Theta \rightarrow \mathbb{R}$ by $U(\mathbf{x}, \theta) \equiv \int u(\mathbf{x}, \mathbf{s}) f(\mathbf{s} ; \theta) d \mu(\mathbf{s})$.

In this context we seek conditions on $u$ and $f$ under which the following monotone comparative statics prediction holds:

(MCS) $\quad \mathbf{x}^{*}(\theta, B) \equiv \arg \max _{x \in B} U(\mathbf{x}, \theta)$ is nondecreasing in $\theta$ and $B$.

In order to make (MCS) precise, we need to specify in what sense $\mathbf{x}^{*}$ should be nondecreasing, as well as what it means for

4. Karlin and Rubin [1956] study the preservation of single-crossing properties under integration with respect to log-spm densities. A number of papers in the statistics literature have exploited this relationship, and Karlin's [1968] monograph presents the general theory of the preservation of an arbitrary number of sign changes under integration. Jewitt [1987] exploits the work on the preservation of single-crossing properties and bivariate log-spm in his analysis of orderings over risk aversion; he makes use of the fact that orderings over risk aversion can be recast as statements about log-spm of a marginal utility.

5. Many authors have studied the comparative statics properties of portfolio and investment problems, including Diamond and Stiglitz [1974], Eeckhoudt and Gollier [1995], Gollier [1995], Hadar and Russell [1978], Jewitt [1986, 1987, 1988b, 1989], Kimball [1990, 1993], Landsberger and Meilijson [1990], Meyer and Ormiston [1983, 1985, 1989], Ormiston [1992], and Ormiston and Schlee [1992, 1993]; see Scarsini [1994] for a survey.

6. Nothing would change in the paper if we let $X$ be an arbitrary lattice; we use $X \subseteq \mathbb{R}^{\mathrm{n}}$ for simplicity.

7. More generally, whenever integrals appear, we assume that requirements of integrability and measurability are met. 
the constraint set $B$ to increase. To do so, we introduce some notation from lattice theory. Given a set $X$ and a partial order $\geq$, the operations "meet" $(\vee)$ and "join" $(\wedge)$ are defined as follows: $\mathbf{x} \vee \mathbf{y}=\inf \{\mathbf{z} \mid \mathbf{z} \geq \mathbf{x}, \mathbf{z} \geq \mathbf{y}\}$ and $\mathbf{x} \wedge \mathbf{y}=\sup \{\mathbf{z} \mid \mathbf{z} \leq \mathbf{x}, \mathbf{z} \leq \mathbf{y}\}$. For $\mathbb{R}^{n}$ with the usual order, these represent the component-wise maximum and component-wise minimum, respectively. A lattice is a set $X$ together with a partial order, such that the set is closed under meet and join.

These definitions can be used to define the order over sets used in (MCS).

Definition 1. A set $A$ is greater than a set $B$ in the strong set order (SSO), written as $A \geq B$, if, for any $a \in A$ and any $b \in$ $B, a \vee b \in A$ and $a \wedge b \in B$. A set-valued function $A: \mathbb{R} \rightarrow$ $2^{\mathbb{R}^{n}}$ is nondecreasing in the strong set order (SSO) if for any $\tau_{H}>\tau_{L}, A\left(\tau_{H}\right) \geq \mathrm{A}\left(\tau_{L}\right)$. A set $A$ is a sublattice if $A \geq A$.

If a set-valued function is nondecreasing in the strong set order, then the lowest and highest elements of this set are nondecreasing. To see an example, a set of the form $\left[a_{1}, b_{1}\right] \times\left[a_{2}, b_{2}\right]$ is nondecreasing (SSO) in $a_{i}$ and $b_{i}, i=1,2$.

We assume throughout that the constraint set $B$ is a sublattice, so that it is closed under the meet and join operations. Thus, if one component of $\mathbf{x}$ increases, the constraint set does not force other components of $\mathbf{x}$ to decrease. Then, Milgrom and Shannon [1994] show that (MCS) holds if and only if $U$ is quasi-supermodular (abbreviated quasi-spm) in $\mathbf{x}$ and satisfies a single crossing property, referred to as $\mathrm{SC} 2$, in $(\mathbf{x} ; \theta) .{ }^{8}$ These properties are defined as follows.

Definition 2. For $T \subseteq \mathbb{R}$, let $g: T \rightarrow \mathbb{R}$. (i) $g$ satisfies single crossing (SC1) in $t$ if there exists inf $T \leq t_{0}^{\prime} \leq t_{0}^{\prime \prime} \leq \sup T$ such that $g(t)<(\leq) 0$ for all $t<(\leq) t_{0}^{\prime}, g(t)=0$ for all $t_{0}^{\prime}<t<t_{0}^{\prime \prime}$, and $g(t)>(\geq) 0$ for all $t>(\geq) t_{0}^{\prime \prime}$.

(ii) $h: \mathbf{X} \times T \rightarrow \mathbb{R}$ satisfies single crossing in two variables (SC2) in $(\mathbf{x} ; T)$ if, for all $\mathbf{x}_{H}>\mathbf{x}_{L}, g(t) \equiv h\left(\mathbf{x}_{H} ; t\right)-h\left(\mathbf{x}_{L} ; t\right)$ satisfies $\mathrm{SC} 1$.

8. Since an empty set is always larger and smaller than any other set in the strong set order, we do not state an assumption about the existence of an optimum (following Milgrom and Shannon [1994]). 
(iii) $h: \mathbf{X} \rightarrow \mathbb{R}$ is quasi-supermodular if it satisfies SC2 in $\left(x_{i} ; x_{j}\right)$ for all $i \neq j .^{9}$

The definition of SC1 simply says that as $t$ increases, $g(t)$ crosses zero at most once and from below. Single crossing in two variables, $\mathrm{SC} 2$, in $(\mathbf{x} ; t)$ requires that the incremental returns to $\mathbf{x}$ satisfy SC1. When $U$ satisfies $\mathrm{SC} 2$ in $(\mathbf{x} ; \theta)$, as $\theta$ increases, an agent choosing between $\mathbf{x}_{L}$ and $\mathbf{x}_{H}$ will first prefer $\mathbf{x}_{L}$, then become indifferent, and finally prefer $\mathbf{x}_{H}$. For establishing (MCS), the additional requirement that $U$ is quasi-spm ensures that increases in the components of $\mathbf{x}$ reinforce one another.

\section{B. Log-Spm Primitives}

To understand when primitive functions might be log-spm, consider first the formal definition. ${ }^{10}$

Definition 3. Let $(X, \geq)$ be a lattice. A function $h: \mathbf{X} \rightarrow \mathfrak{R}$ is supermodular if, for all $\mathbf{x}, \mathbf{y} \in X, h(\mathbf{x} \vee \mathbf{y})+h(\mathbf{x} \wedge \mathrm{y}) \geq$ $h(\mathbf{x})+h(\mathbf{y}) . h$ is log-supermodular (log-spm) if it is nonnegative, and, for all $\mathbf{x}, \mathbf{y} \in X, h(\mathbf{x} \vee \mathbf{y}) \cdot h(\mathbf{x} \wedge \mathbf{y}) \geq h(\mathbf{x}) \cdot h(\mathbf{y})$.

To interpret this, observe that for a function $h: \mathbb{R}^{2} \rightarrow \mathbb{R}$, supermodularity requires that the incremental returns to increasing $x$, defined by $g(t) \equiv h\left(x_{H} ; t\right)-h\left(x_{L} ; t\right)$, must be nondecreasing in $t$; log-supermodularity of a positive function requires that the relative returns, $h\left(x_{H} ; t\right) / h\left(x_{L} ; t\right)$, are nondecreasing in $t$. The multivariate version of supermodularity simply requires that the relationship just described holds for each pair of variables. ${ }^{11}$ Topkis [1978] proves that if $h$ is twice differentiable, $h$ is supermodular if and only if $\left(\partial^{2} / \partial x_{i} \partial x_{j}\right) h(\mathbf{x}) \geq 0$ for all $i \neq j$. If $h$ is positive, then $h$ is log-spm if and only if $\log (h(\mathbf{x}))$ is supermodular. Both properties are stronger than quasi supermodularity; thus, as properties of objective functions, both are sufficient for comparative statics predictions to hold. Observe that sums of supermodular functions are supermodular, and products of log-

9. This definition applies because we assumed that $X$ is a product set. When $X$ is an arbitrary lattice, $h$ is quasi-supermodular if for all $\mathbf{x}, \mathbf{y} \in X, h(\mathbf{x})-h(\mathbf{x} \wedge$ y) $\geq(>) 0$ implies $h(\mathbf{x} \vee \mathbf{y})-h(\mathbf{y}) \geq(>) 0$.

10. Karlin and Rinott [1980] referred to log-spm as multivariate total positivity of order 2.

11. Although supermodularity can be checked pairwise (see Topkis [1978]), Lorentz [1953] and Perlman and Olkin [1980] establish that the pairwise characterization of log-spm requires additional assumptions, such as strict positivity (at least throughout "order intervals"). 
spm functions are log-spm. However, sums of log-spm functions are not necessarily log-spm.

Consider some examples where economic primitives are logspm. A parameterized demand function $D(P ; t)$ is log-spm if and only if the price elasticity, $\epsilon(P ; t) \equiv P \cdot D_{P}(P ; t) / D(P ; t)$, is nondecreasing in $t$. A marginal utility function $U^{\prime}(w+s)$ is log-spm in $(w, s)$ (where $w$ often represents initial wealth and $s$ represents the return to a risky asset) if and only if the utility satisfies decreasing absolute risk aversion (DARA). A parameterized distribution $F(\cdot ; \theta)$ has a hazard rate $f(s ; \theta) /(1-F(s ; \theta))$ which is nonincreasing in $\theta$ if $1-F(s ; \theta)$ is log-spm. Milgrom and Weber [1982] show that a vector of random variables is affiliated if and only if their joint density is log-spm (almost everywhere). When the support of $F(\cdot ; \theta)$, denoted supp $[F]$, is constant in $\theta$, and $F$ has a density $f,{ }^{12}$ then the Monotone Likelihood Ratio Order (MLR) requires that $f$ is $\log$-spm, that is, the likelihood ratio $f\left(s ; \theta_{H}\right) /$ $f\left(s ; \theta_{L}\right)$ is nondecreasing in $s$ for all $\theta_{H}>\theta_{L}{ }^{13}$

\section{Necessary and Sufficient Conditions for Comparative Statics}

Our first step in analyzing (MCS) for problems with log-spm primitives follows.

Lemma 1. Suppose that $f$ is nonnegative. Then (i) (MCS) holds for all $u: X \times S \rightarrow \mathbb{R}_{+}$log-spm, if and only if (ii) $U$ is log-spm in $(\mathbf{x}, \theta)$ for all $u: X \times S \rightarrow \mathbb{R}_{+}$log-spm.

Lemma 1 states that if we want (MCS) to hold for all log-spm payoffs, the objective function $U$ must be log-spm (a stronger condition than quasi-spm). The proof shows that if $U$ fails to be log-spm in $(\mathbf{x}, \theta)$ for some $u$ log-spm, then we can find another $\log$-spm payoff $v$, for which (MCS) fails. Lemma 1 then motivates the main technical question for this section: under what conditions on $f$ does log-supermodularity of $u$ imply that $U$ is log-spm? Consider a result from the statistics literature, which will be one of the main tools used in this paper.

Lemma 2 [Ahlswede and Daykin 1979]. Let $h_{i}(i=1, \ldots, 4)$ represent four nonnegative functions, $h_{i}: S \rightarrow \mathbb{R}$. Then condition (L2.1) implies (L2.2):

12. The MLR can also be defined for distributions with varying supports, or distributions that do not have densities with respect to Lebesgue measure; see the Appendix for details.

13. See Lehmann [1955] and Milgrom [1981]. Related, in the statistics literature [Karlin 1968], a strictly positive bivariate function satisfies total positivity of order 2 (TP-2) if and only if it is log-spm. 
(L2.1)

$$
h_{1}(\mathbf{s}) \cdot h_{2}\left(\mathbf{s}^{\prime}\right) \leq h_{3}\left(\mathbf{s} \vee \mathbf{s}^{\prime}\right) \cdot h_{4}\left(\mathbf{s} \wedge \mathbf{s}^{\prime}\right) \text { for } \mu \text {-almost all } \mathbf{s}, \mathbf{s}^{\prime} \in \mathrm{S} \text {. }
$$

$$
\int h_{1}(\mathbf{s}) d \mu(\mathbf{s}) \cdot \int h_{2}(\mathbf{s}) d \mu(\mathbf{s}) \leq \int h_{3}(\mathbf{s}) d \mu(\mathbf{s}) \cdot \int h_{4}(\mathbf{s}) d \mu(\mathbf{s}) .
$$

Karlin and Rinott [1980] provide a simple proof of this lemma. ${ }^{14}$ They further explore a variety of interesting applications in statistics, although they do not consider the problem of comparative statics. While we will use this result in a variety of ways throughout the paper, the most important (and immediate) consequence of Lemma 2 for comparative statics is that log-supermodularity is preserved by integration. To see this, set

$$
\begin{gathered}
h_{1}(\mathbf{s})=g(\mathbf{y}, \mathbf{s}), \quad h_{2}(\mathbf{s})=g\left(\mathbf{y}^{\prime}, \mathbf{s}\right), \quad h_{3}(\mathbf{s})=g\left(\mathbf{y} \vee \mathbf{y}^{\prime}, \mathbf{s}\right), \\
\text { and } h_{4}(\mathbf{s})=g\left(\mathbf{y} \wedge \mathbf{y}^{\prime}, \mathbf{s}\right) .
\end{gathered}
$$

Then (L2.1) states exactly that $g(\mathbf{y}, \mathbf{s})$ is log-spm in $(\mathbf{y}, \mathbf{s})$, while (L2.2) reduces to the conclusion is that $\int g(\mathbf{y}, \mathbf{s}) d \mu(\mathbf{s})$ is log-spm in y. Recall that arbitrary sums of log-spm functions are not logspm, which makes this result somewhat surprising. But notice that Lemma 2 does not apply to arbitrary sums, only to sums of the form $g\left(\mathbf{y}, \mathbf{s}^{1}\right)+g\left(\mathbf{y}, \mathbf{s}^{2}\right)$, when $g$ is log-spm in all arguments.

The preservation of log-spm under integration is especially useful for analyzing expected values of payoff functions. Since arbitrary products of log-spm functions are log-spm, a sufficient condition for $\int u(\mathbf{x}, \mathbf{s}) f(\mathbf{s} ; \theta) d \mu(\mathbf{s})$ to be log-spm is that $u$ and $f$ are log-spm. To understand the intuition, consider first the case where $X, S \subseteq \mathbb{R}$. Then, $u$ is log-spm implies that the relative returns to $x$ are nondecreasing in $s$. If $f$ is log-spm, then $\theta$ increases the likelihood of high values of $s$ relative to low values of $s$. Then, Lemma 2 implies that $\theta$ increases the expected relative returns to $x$. In the multivariate case, $\log$-spm of $u$ and $f$ ensure that the interactions among components of $\mathbf{x}$ and $\mathbf{s}$ reinforce these

14. This result has a long history in statistics. Lehmann [1955] proves that bivariate log-supermodularity is preserved by integration. Ahlswede and Daykin's [1979] result essentially extends the theory to multivariate functions. Karlin and Rinott [1980] present the theory of multivariate total positivity of order 2 (MTP-2) functions together with a variety of applications in statistics. Milgrom and Weber [1982] independently derive a variety of properties of affiliated random variables in auctions. See also Whitt [1982]. 
effects. Karlin and Rinott [1980] give many examples of densities that are log-spm, and thus preserve log-spm of a payoff function. ${ }^{15}$ Below, in subsection II.D, we show how the result can be used in analyzing the preservation of ratio orderings, such as decreasing absolute risk aversion.

An especially important type of log-spm function for our purposes is an indicator function for a sublattice or a nondecreasing set-valued function.

Lemma 3 . Given $A: \mathbb{R} \rightarrow 2^{S}$, the indicator function $1_{A(\tau)}(\mathbf{s})$ is $\log$-spm in $(\mathbf{s}, \tau)$ if and only if $A(\tau)$ is a sublattice for each $\tau$, and is nondecreasing (strong set order).

Lemma 3 implies, for example, that $1_{[a, b]}(s)$ is log-spm in $(a, b, s)$. To see how Lemma 3 can be used, observe that Lemmas 2 and $3 \mathrm{imply}$ log-spm of a distribution is weaker than log-spm of a density. Formally, if $f(s ; \theta)$ is log-spm (that is, the distribution satisfies the MLR order), then the corresponding cumulative distribution function $F(s ; \theta)=\int 1_{(-\infty, s]}(t) f(t ; \theta) d t$ will be log-spm (that is, it satisfies the Monotone Probability Ratio Order), ${ }^{16}$ which can be shown to be stronger than First Order Stochastic Dominance. This in turn implies that $\int_{-\infty}^{a} F(s ; \theta) d s$ is log-spm, which is stronger than Second Order Stochastic Dominance if $\theta$ does not change the mean of $s$.

In a similar way, Lemma 3 can also be used to show that $\log$-spm of $f$ is a necessary condition for $U$ to be log-spm whenever $u$ is log-spm. Lemma 3 helps us identify a set of "test functions" that are a subset of the set of log-spm functions; these are the functions used to create counterexamples. Consider the special case where $X=S$. Define $B_{\epsilon}(\mathbf{x})=\left\{\mathbf{y} \in X: \forall i, y_{i} \in\left[x_{i}-\epsilon, x_{i}+\epsilon\right]\right\}$. Because for each $\mathbf{x}$ and $\epsilon, B_{\epsilon}(\mathbf{x})$ is a sublattice and is nondecreasing in $\mathbf{x}$ in the strong set order, Lemma 3 implies that $1_{B_{\epsilon}(\mathbf{x})}(\mathbf{s})$ is $\log$-spm in $(\mathbf{x}, \mathbf{s})$. Define a set of such functions as follows:

$$
\boldsymbol{\gamma}(\beta) \equiv\left\{u: \exists 0 \leq \epsilon<\beta \text { such that } u(\mathrm{x}, \mathrm{s}) \equiv 1_{B_{\epsilon}(x)}(\mathbf{s})\right\} .
$$

Then, for any $\beta>0, U$ is log-spm in $(\mathbf{x}, \theta)$ whenever $u \in \boldsymbol{\gamma}(\beta)$, if

15. For example, exchangeable, positively correlated normal vectors and absolute values of normal random vectors have log-supermodular densities (but arbitrary positively correlated normal random vectors do not; Karlin and Rinott [1980] give restrictions on the covariance matrix which suffice); and multivariate logistic, $F$, and gamma distributions have log-supermodular densities.

16. See Eeckhoudt and Gollier [1995], who show that an MPR shift is sufficient for a risk-averse investor to increase his portfolio allocation. 
and only if $f$ is log-spm in $(\mathbf{s}, \theta)$ a.e. $-\mu .{ }^{17}$ To see this, let $\mu$ be Lebesgue, and suppose that $f$ is continuous. Then $\lim _{\epsilon \rightarrow 0}$ $\int 1_{B_{\epsilon}(x)}(\mathbf{s}) f(\mathbf{s} ; \theta) d \mu(\mathbf{s})=f(\mathbf{x} ; \theta)$. This formalizes what we mean by a set of test functions: $\gamma(\beta)$ is a subset of log-spm functions, but the set is large enough to make log-spm of $f$ a necessary condition for $U$ to be log-spm. ${ }^{18}$ The following result generalizes this discussion.

Lemma 4. Suppose that $f$ is nonnegative, and that $n \geq 2$ if $m \geq 2$ (where $m$ is the dimension of $S$ and $n$ is the dimension of $X$ ). The following two conditions are equivalent: (i) $U$ is log-spm in $(\mathbf{x}, \theta)$ for all $u: X \times S \rightarrow \mathbb{R}_{+}$that are log-spm a.e.- $\mu$; (ii) $f$ is $\log$-spm in $(\mathbf{s}, \theta)$ a.e.- $\mu$.

REMARK. Lemma 4 requires that $\mathbf{x}$ has at least two components $(n \geq 2)$ if $\mathrm{s}$ has two or more components $(m \geq 2) .{ }^{19}$ However, even in cases where $m \geq 2$ and $n=1$, (ii) is sufficient for (i), and further, log-supermodularity of $f$ in $\left(s_{i}, \theta\right)$ for all $i$ is necessary for (i) to hold.

Lemma 4 is of interest in its own right, as we will see below. For the moment, however, we use Lemma 4 (together with Lemma 1) to prove our first comparative statics theorem.

Theorem 1 (Comparative Statics with Log-Supermodular Primitives). Suppose that $f$ is nonnegative, and suppose that $n \geq 2$ if $m \geq 2$ (where, again, $m$ is the dimension of $S$ and $n$ is the dimension of $X$ ). The following two conditions are equivalent: (i) (MCS) holds for all $u: X \times S \rightarrow \mathbb{R}_{+}$that are log-spm a.e.- $\mu$; (ii) $f$ is $\log$-spm in $(\mathbf{s}, \theta)$ a.e. $-\mu$.

Theorem 1 gives necessary and sufficient conditions for comparative statics when the payoff function is log-spm. Further, the

17. We say that $f$ is $\log$-spm a.e.- $\mu$ if the inequality in Definition 3 holds for almost all (with respect to the product measure on $S \times S$ induced by $\mu$ ) $\left(\mathbf{s}, \mathbf{s}^{\prime}\right)$ pairs in $S \times S$.

18. Our approach to necessity can be understood with reference to the stochastic dominance literature, and more generally Athey [1998]. Athey [1998] shows that in stochastic problems, if one wishes to establish that a property $P$ holds for $U(\mathbf{x}, \theta)$ for all $u$ in a given class $u$, it is often necessary and sufficient to check that $P$ holds for all $u$ in the set of extreme points of $u$. The extreme points can be thought of as test functions; for example, when $\boldsymbol{U}$ is the set of nondecreasing functions, the set of test functions is the set of indicator functions for sets $1_{\mathbf{A}}(\mathbf{s})$ that are nondecreasing in $\mathbf{s}$. Athey [1998] shows that this approach works well when $P$ is a property preserved by convex combinations, unlike log-supermodularity.

19. If we extend the analysis so that $X$ is an arbitrary lattice rather than a product set, we require that $X$ has at least two elements that are not ordered. 
approach based on test functions allows the modeler to immediately check whether any additional restrictions on the class of admissible payoff functions (where such restrictions might be motivated by an economic model) affect the necessity conclusion. For example, the elements of $7(\beta)$ are clearly not monotonic, and thus the "only if" part of Theorem 1 does not hold under the additional assumption that $u$ is nondecreasing. In contrast, we can approximate the elements of $\chi \beta$ ) with smooth functions, so smoothness restrictions will not alter the conclusion of Theorem 1.

Finally, we observe that the results of Lemmas 2 and 3 can be combined to provide sufficient conditions for comparative statics in problems outside the particular structure considered in (MCS). For example, suppose that for $j=1, \ldots, J, A^{j}: \mathbb{R} \rightarrow 2^{S}, A^{j}\left(\tau_{j}\right)$ is a sublattice and is nondecreasing in $\tau_{j}$, and let $\mathrm{A}(\tau)=\bigcap_{j=1, \ldots, J}$ $A^{j}\left(\tau_{j}\right)$. Then if $g$ and $h$ are $\log$-spm, $\mathbf{x}^{*}(\theta, \tau, B)=\arg \max _{\mathbf{x} \in B} \int_{\mathbf{s} \in \mathbf{A}(\tau)}$ $g(\mathbf{x}, \theta, \mathbf{s}) h(\mathbf{s} ; \mathbf{x}, \theta) d \mu(\mathbf{s})$ is nondecreasing in all of its arguments. ${ }^{20}$

\section{Applications}

The first set of applications concerns risk aversion and background risks. Lemma 4 can be applied to problems that involve orderings of ratios. For example, consider $g, h: \mathbb{R} \rightarrow \mathbb{R}_{+}$. Then, define $u:\{0,1\} \times \mathbb{R} \rightarrow \mathbb{R}_{+}$by $u(x, s)=g(s)$ if $x=1$ and $u(x, s)=h(s)$ if $x=0$. Now observe that if $h>0, u$ is log-spm if and only if $g(s) / h(s)$ is nondecreasing in $s$. Using this construction, Lemma 4 implies that $\int g(s) f(s ; \theta) d s / \int h(s) f(s ; \theta) d s$ is nondecreasing in $\theta$ for all $g, h: \mathbb{R} \rightarrow \mathbb{R}_{+}$such that $g(s) / h(s)$ is nondecreasing in $s$, if and only if $f$ is log-spm in $(s ; \theta)$.

The Arrow-Pratt coefficient of absolute risk aversion is defined in terms of a ratio. Let the coefficient of risk aversion of the utility function $u$ evaluated at $w$ be $R(w ; u(\cdot)) \equiv-u^{\prime \prime}(w) / u^{\prime}(w)$, and let $U(w ; \gamma, \theta) \equiv \int u(w+s ; \gamma) f(s ; \theta) d s$. Then, Lemma 4 has the following implication: ${ }^{21}$

20. However, note that even if $h$ is a probability density, the objective function in this problem cannot be interpreted as a conditional expectation of $g$, since we have not divided by the probability that $\mathbf{s} \in A(\tau)$. The working paper [Athey 1996] uses Lemmas 2 and 3 to provide necessary and sufficient conditions for comparative statics predictions to hold when the objective function is a conditional expectation.

21. Pratt [1988] first established that risk aversion orderings are preserved by expectations, while Jewitt [1987] first showed that the "more risk averse" ordering is preserved by a MLR shift. The results about prudence are new. 
1. MLR Shifts and Decreasing Absolute Risk Aversion. If $R(w ; u(\cdot ; \gamma))$ is nonincreasing in $w$ and $f$ is $\log$-spm in $(s, \theta)$, then $R(w ; U(\cdot ; \gamma, \theta))$ is nonincreasing in $\theta$.

Following similar logic, Lemma 4 can be used to show

2. Preservation of Decreasing Absolute Risk Aversion under Background Risks. If $R(w ; u(\cdot ; \gamma))$ is nonincreasing in $w$ and $\gamma$, then $R(w ; U(\cdot ; \gamma, \theta))$ is nonincreasing in $w$ and $\gamma .{ }^{22}$

3. Prudence. Results (1) and (2) hold for a risk-averse agent when $R(w ; u)$ is replaced by prudence [Kimball 1990], $P(w$; $u(\cdot)) \equiv-u^{\prime \prime \prime}(w) / u^{\prime \prime}(w)$, if $u^{\prime \prime \prime}>0$ (or $-P$, if $u^{\prime \prime \prime}<0$ ).

Lemma 4 can also be used to analyze the average risk aversion of a group of agents; suppose, for example, that a retirement fund manager seeks to maximize the average utility of a group of workers with heterogeneous portfolios $(\theta)$ and risk preferences $(\gamma)$ :

4. Populations of Agents. Consider a group of agents as above. Let $h(\theta, \gamma ; \eta)$ be the joint density of $(\theta, \gamma)$ in the population. Let $\bar{U}(w ; \eta)=\iint U(w ; \gamma, \theta) h(\theta, \gamma ; \eta) d \theta d \gamma$ be the average expected utility for the population. If $R(w ; u(\cdot ; \gamma))$ is nonincreasing in $w$ and $\gamma$, and $f$ and $h$ are log-spm, then $R(w ; \bar{U}(\cdot ; \eta))$ is nonincreasing in $w$ and $\eta$. In words, if higher (MLR) distributions of investment returns are affiliated with lower risk aversion parameters, then the average utility satisfies DARA, and an MLR shift in the distribution of $\theta$ or $\gamma$ decreases population risk aversion.

A second set of applications concerns log-spm games of incomplete information. Consider a game of incomplete information where each player has private information about her own type, $t_{i} \in T_{i} \subseteq \mathbb{R}$, and chooses a strategy $\chi_{i}: T_{i} \rightarrow X_{i}$, where $X_{i} \subseteq \mathbb{R}$. Athey [2001] shows that a pure strategy Nash equilibrium (PSNE) in nondecreasing strategies exists in games of incomplete information where an individual player's strategy $\chi_{i}(\cdot)$ is nondecreasing in her type, whenever all of her opponents use nondecreasing strategies. ${ }^{23}$

22. To see this, observe that $u^{\prime}(w+s ; \gamma)$ is log-spm in $(w, s, \gamma)$ if and only if $u^{\prime \prime}(w+s ; \gamma) / u^{\prime}(w+s ; \gamma)$ is nondecreasing in $w, s$, and $\gamma$, which is in turn equivalent to requiring that $R(w ; u(\cdot ; \gamma))$ is nonincreasing in $w$ and $\gamma$. This implies that $u^{\prime}(w+$ $s ; \gamma) f(s)$ is log-spm in $(w, s, \gamma)$, and the result follows by Lemma 4.

23. This result requires that the type distribution is atomless (Lebesgue), and that either $X$ is finite, or $X$ is compact and convex and payoffs have only certain types of discontinuities. This result is distinct from results based on the theory of supermodular games. For example, Vives [1990] analyzes games where increase in an opponent's strategy leads to a pointwise increase in a player's best response. This in turn implies that a PSNE exists. In contrast, if each player's payoff $v_{i}(\mathbf{x})$ is log-spm, the game does not necessarily have strategic complementarities, and a different approach is required. 
Let $T=T_{1} \times \cdots \times T_{I}$, let $h: \mathrm{T} \rightarrow \mathbb{R}_{+}$be the joint density over types (with respect to Lebesgue measure), assumed strictly positive on $T$, and let $h_{i}\left(\cdot \mid t_{i}\right)$ be the conditional density of player $i$ 's opponents' types. Let player $i$ 's utility be given by $v_{i}: X \times T \rightarrow \mathbb{R}$. Expected payoffs are $V_{i}\left(x_{i}, t_{i}\right) \equiv \int_{\mathbf{t}_{-i}} v_{i}\left(x_{i}, \chi_{-i}\left(\mathbf{t}_{-i}\right), \mathbf{t}\right) h_{-i}\left(\mathbf{t}_{-i} \mid t_{i}\right) d \mathbf{t}_{-i}$. The following result gives necessary and sufficient conditions for each player's best response to nondecreasing strategies to be nondecreasing.

Proposition 1. The following two conditions are equivalent: (i) for all $i, \chi_{i}\left(t_{i}\right) \equiv \arg \max _{x_{i}} V_{i}\left(x_{i}, t_{i}\right)$ is nondecreasing in $t_{i}$ for all $\chi_{j}(\cdot)$ nondecreasing for $j \neq i$, and all $v_{i} \log$-spm; (ii) the types are affiliated.

Spulber [1995] analyzed how asymmetric information about a firm's cost parameters alters the results of a Bertrand pricing model, showing that there exists an equilibrium where prices are increasing in costs, and further firms price above marginal cost and have positive expected profits. Spulber's model assumes that costs are independently and identically distributed; Proposition 1 generalizes his result to asymmetric, affiliated signals, and to imperfect substitutes. To see this, let $v_{i}(\mathbf{x}, \mathbf{t})=\left(x_{i}-t_{i}\right) D_{i}(\mathbf{x})$, where $\mathbf{x}$ is the vector of prices, $\mathbf{t}$ is the vector of marginal costs, and $D_{i}(\mathbf{x})$ gives demand to firm $i$ when prices are $\mathbf{x}$. First observe that $x_{i}-$ $t_{i}$ is $\log$-spm. Then, by Lemma 4 , the expected payoff function is log-spm if the signals are affiliated, each opponent uses a nondecreasing strategy, and $D_{i}(\mathbf{x})$ is log-spm. The interpretation of the latter condition is that the elasticity of demand is a nonincreasing function of the other firms' prices. ${ }^{24}$ When the goods are perfect substitutes, expected demand is given by (ignoring ties for simplicity)

$$
D_{1}\left(x_{1}\right) \int_{t_{-1}} 1_{\chi_{2}\left(t_{2}\right)>x_{1}}\left(t_{2}\right) \cdots 1_{\chi_{n}\left(t_{n}\right)>x_{1}}\left(t_{2}\right) h\left(\mathbf{t}_{-1} \mid t_{1}\right) d \mathbf{t}_{-1} .
$$

Since the set $\left\{t_{j}: \chi_{j}\left(t_{j}\right)>x_{1}\right\}$ is nondecreasing (strong set order) in $x_{1}$ when each $\chi_{i}$ is nondecreasing by Lemma 3, expected payoffs must be log-spm when the density $h$ is log-spm.

24. Demand functions that satisfy these criteria include logit, CES, transcendental logarithmic, and a set of linear demand functions (see Milgrom and Roberts [1990b] and Topkis [1979]). 


\section{Comparative Statics with Single-Crossing Primitives}

This section studies single-crossing properties in problems where there is a single real-valued random variable. Multivariate generalizations of the results are sufficiently restrictive that they are not considered here. However, many problems in the theory of investment under uncertainty concern a single random variable, and a number of other problems (such as auctions) can be reformulated so that the techniques from this section apply.

Formally, in this section we assume that $S \subseteq \mathbb{R}$, and for simplicity we also assume that $X \subseteq \mathbb{R}$. Then, we define $U(x, \theta) \equiv$ $\int u(x, s) f(s ; \theta) d \mu(s)$. Although the results of Section II apply to this problem, in this section we seek to relax the assumption that both primitives are log-spm. In particular, we consider the weaker assumption that $u(x, s)$ satisfies SC2 in $(x ; s)$. Our comparative statics question becomes to find conditions on $u$ and $f$ under which

$\left(\mathrm{MCS}^{\prime}\right) \quad x^{*}(\theta, B)=\arg \max _{x \in B} U(x, \theta)$ is nondecreasing in $\theta$ and $B$.

Theorem 1 gives some initial insight into this problem: since the set of SC2 functions includes the set of log-spm functions, Theorem 1 implies that a necessary condition for $\left(\mathrm{MCS}^{\prime}\right)$ to hold for all $u$ which are SC2 is that $f$ is log-spm. a.e.- $\mu$. However, we have not established that $u$ SC2 and $f$ log-spm are sufficient for $\left(\mathrm{MCS}^{\prime}\right)$, nor have we addressed the question of what conditions on $u$ are necessary for the conclusion that $\left(\mathrm{MCS}^{\prime}\right)$ holds for all $f$ log-spm.

Before proceeding, we introduce a definition that will allow us to state concisely the results in this section. The definition helps to state theorems about pairs of hypotheses about $u$ and $f$ which guarantee a monotone comparative statics conclusion.

Definition 4. Two hypotheses $H-A$ and $H-B$ are a minimal pair of sufficient conditions (MPSC) for the conclusion $C$ if (i) $C$ holds whenever $H$ - $B$ does, if and only if $H$-A holds. (ii) $C$ holds whenever $H$ - $A$ does, if and only if $H-B$ holds.

This definition captures the idea that we are looking for a pair of sufficient conditions that cannot be weakened without placing further structure on the problem. In some contexts, $H-A$ will be given (such as an assumption on $u$ ), and we will search for the weakest hypothesis $H-B$ (such as an assumption on $f$ ) that preserves the conclusion; in other problems, the roles of $H-A$ and 
$H$ - $B$ will be reversed. The definition can be used to state this section's main comparative statics result.

Theorem 2 (Comparative Statics with Single-Crossing Payoffs). (A) $u$ satisfies $\mathrm{SC} 2$ in $(x ; s)$ a.e.- $\mu$; and (B) $f$ is log-spm a.e. $-\mu$; are a MPSC for (C) $\left(\mathrm{MSC}^{\prime}\right)$ holds. $^{25}$

Theorem 2 has an interesting interpretation. Recall that SC2 of $u(x, s)$ (condition T2-A) is the necessary and sufficient condition for the choice of $x$ which maximizes $u(x, s)$ (under certainty) to be nondecreasing in $s$. Thus, Theorem 2 gives necessary and sufficient conditions for the preservation of comparative statics results under uncertainty. ${ }^{26}$ Any result that holds when $s$ is known, will hold when $s$ is unknown but the distribution of $s$ experiences an MLR shift. Further, MLR shifts are the weakest distributional shifts that guarantee that conclusion. In Section IV we illustrate in applications how additional commonly encountered restrictions on $u$ or $f$ can be used to relax (T2-A) and (T2-B).

We outline the proof of Theorem 2 in the text. Our first step is to transform the problem by taking the first difference with respect to $x$. In particular, $U(x, \theta)$ and $u(x, s)$ satisfy SC2 (in $(x ; \theta)$ and $(x ; s))$ if and only if, for all $x_{H}>x_{L}, U\left(x_{H}, \theta\right)-U\left(x_{L}, \theta\right)$ and $u\left(x_{H}, s\right)-u\left(x_{L}, s\right)$ satisfy SC1 (in $\theta$ and $s$ ). The following lemma characterizes SC1 for problems with a single random variable. ${ }^{27}$ It is stated in more general notation because not only will we apply it in cases where $u$ is a payoff function and $f$ is a probability density, but also we will apply it to transformed objective functions (where for example, $f$ is a probability distribution) as well as

25. The quantification over constraint sets $B$ in $\left(\mathrm{MCS}^{\prime}\right)$ can be dropped for the result that (B) is necessary for $(\mathrm{C})$ to hold whenever (A) does.

26. Jewitt [1987] and Ormiston and Schlee [1993] also give this interpretation in their analyses. Ormiston and Schlee explicitly analyze the preservation of comparative statics with respect to MLR shifts, and further show, under additional regularity assumptions, that single crossing of $u$ is a necessary condition for the result to hold for all MLR shifts.

27 . The theory of the preservation of single-crossing properties under uncertainty has been studied by a number of authors in the statistics literature. Karlin and Rubin [1956] establish sufficiency, and Karlin [1968, pp. 233-237] analyzes necessary conditions, under some additional regularity conditions (including the assumption that $\Theta$ has at least three points; Karlin's proof is designed to solve a more complicated problem and thus requires an elaborate construction). In Extension (ii) to Lemma 5 below, we relax Karlin's maintained assumptions about absolute continuity. When $K$ is a probability distribution, the maintained absolute continuity assumption may be undesirable; however, if $k$ represents a utility function, it is the right assumption. 
in problems where the agent's choice variable affects the probability distribution directly.

Lemma 5. Let $g: S \rightarrow \mathbb{R}$ and $k: S \times \Theta \rightarrow \mathbb{R}$. (A) $g$ satisfies SC1 a.e. $-\mu ;{ }^{28}$ and (B) $k$ is log-spm a.e.- $\mu$; are a MPSC for (C) $G(\theta) \equiv$ $\int g(s) k(s ; \theta) d \mu(s)$ satisfies $\mathrm{SC} 1$.

Several extensions to Lemma 5 will be useful in our applications. To state them, we need another definition: we say that $g$ : $\mathbb{R} \rightarrow \mathbb{R}$ satisfies weak SC1 if there exists a $t_{0}$ such that $g(t) \leq 0$ for all $t<t_{0}$ and $g(t) \geq 0$ for all $t>t_{0}$, while $h: \mathbf{X} \times \mathbb{R} \rightarrow \mathbb{R}$ satisfies weak SC2 in $(\mathbf{x} ; t)$ if, for all $\mathbf{x}_{H}>\mathbf{x}_{L}, g(t) \equiv h\left(\mathbf{x}_{H} ; t\right)-h\left(\mathbf{x}_{L} ; t\right)$ satisfies weak SC1.

Extensions to Lemma 5. ${ }^{29}$ Lemma 5 also holds under any of the following modifications: (i) $g$ depends on $\theta$ directly, under the additional restrictions that $g$ is piecewise continuous in $\theta$ and either (a) $g$ is nondecreasing in $\theta$, or (b) for all $\theta, g$ is nonzero except at a single (fixed) point $s_{0}$, and further, for all $\theta_{H}>\theta_{L}$, $g\left(s, \theta_{H}\right) / g\left(s, \theta_{L}\right)$ is nondecreasing in $s$.

(ii) We allow that for each $\theta$, there exists a measure $\mu^{\theta}$ such that $K(s ; \theta) \equiv \int_{-\infty}^{s} k(t, \theta) d \mu^{\theta}(t)$, we define $G(\theta) \equiv \int g(s)$ $d K(s ; \theta)$, and we replace $(\mathrm{B})$ with $\left(\mathrm{B}^{\prime}\right) \theta$ orders $K(\cdot ; \theta)$ by MLR.

(iii) supp $[K(\cdot ; \theta)]$ is constant in $\theta$, and (A) is replaced with $\left(\mathrm{A}^{\prime}\right) \mathrm{g}$ satisfies weak SC1.

Consider first sufficiency. Intuitively, if $g$ satisfies SC1 and crosses zero at $s_{0}$, then log-spm of $k$ guarantees that as $\theta$ increases, the weight on $s$ relative to $s_{0}$ increases (decreases) for values of $s$ where $g$ is nonnegative (nonpositive), i.e., where $s>$ $(<) s_{0}$. More formally, suppose for simplicity that $k>0$. Define $l(s) \equiv k\left(s ; \theta_{H}\right) / k\left(s ; \theta_{L}\right)$. (L5-B) implies that $l(\cdot)$ is nondecreasing. In turn, this implies that for every possible crossing point $s_{0}$ :

$$
l(s) \leq l\left(s_{0}\right) \quad \text { for } s<s_{0}, \quad \text { while } l(s) \geq l\left(s_{0}\right) \quad \text { for } s>s_{0} .
$$

Condition (1) is then used to establish sufficiency, as follows. Given $\theta_{H}>\theta_{L}$ and a point $s_{0}$ where $g$ crosses zero,

28. We will say that $g(s)$ satisfies SC1 almost everywhere- $\mu$ (a.e.- $\mu$ ) if conditions in the definition of SC1 hold for almost all (with respect to the product measure on $S \times S$ induced by $\mu)\left(s_{L}, s_{H}\right)$ pairs in $S \times S$ such that $s_{L}<s_{H}$.

29. A version of this theorem which gives minimal sufficient conditions for strict single crossing is provided in Athey [1996]. 
(2)

$$
\begin{aligned}
& \int g(s) k\left(s ; \theta_{H}\right) d \mu(s) \\
& =\int g(s) l(s) k\left(s ; \theta_{L}\right) d \mu(s) \\
& =-\int_{-\infty}^{s_{0}}|g(s)| l(s) k\left(s ; \theta_{L}\right) d \mu(s)+\int_{s_{0}}^{\infty} g(s) l(s) k\left(s ; \theta_{L}\right) d \mu(s) \\
& \geq-l\left(s_{0}\right) \int_{-\infty}^{s_{0}}|g(s)| k\left(s ; \theta_{L}\right) d \mu(s)+l\left(s_{0}\right) \int_{s_{0}}^{\infty} g(s) k\left(s ; \theta_{L}\right) d \mu(s) \\
& =l\left(s_{0}\right) \int g(s) k\left(s ; \theta_{L}\right) d \mu(s) .
\end{aligned}
$$

The second equality holds because $g$ satisfies SC1, while the inequality follows from (1). If, in addition, $l\left(s_{0}\right) \leq 1$, then $\int g(s) k(s$; $\left.\theta_{H}\right) d \mu(s) \leq 0$ implies that $\int g(s) k\left(s ; \theta_{L}\right) d \mu(s) \leq \int g(s) k\left(s ; \theta_{H}\right) d \mu(s)$.

The necessity parts of this theorem can be proved by constructing counterexamples, which are drawn from an appropriate set of test functions:

Lemma 6 (Test Functions for Single-Crossing Problems). Define the following sets:

$$
\begin{gathered}
\mathcal{g}(\beta) \equiv\left\{g: \exists a, b>0, \beta>\epsilon, \delta>0, \text { and } s_{L}<s_{H} \text { such that } g(s)\right. \\
=-a \text { for } s \in\left(s_{L}-\epsilon, s_{L}+\epsilon\right), g(s)=b \text { for } s \in \\
\left.\left(s_{H}-\epsilon, s_{H}+\epsilon\right)|g|<\delta \text { elsewhere, and } g \text { satisfies SC1 }\right\} \\
\mathbb{X}(\beta) \equiv\{k: \exists a>0 \text { and } h: \Theta \rightarrow S, h \text { increasing, and some } \\
\beta>\epsilon>0 \text { such that } k(s, \theta)=a \text { for } s \in(h(\theta)-\epsilon, \\
h(\theta)+\epsilon), \text { and } k(s, \theta)=0 \text { elsewhere }\} .
\end{gathered}
$$

Then Lemma 5 holds if, for any $\beta>0$, (L5-A) is replaced with $\left(\mathrm{L} 5-\mathrm{A}^{\prime}\right) \mathrm{g} \in \mathcal{G}(\beta)$; Lemma 5 also holds if (L5-B) is replaced with $\left(\mathrm{L} 5-\mathrm{B}^{\prime}\right) k \in \mathbb{R}(\beta)$.

As in Lemma 4, placing smoothness assumptions on $g$ or $k$ will not change the conclusions of Lemmas 5 and 6 , but monotonicity or curvature assumptions will. 
Now consider how the counterexamples are used. If $g$ fails (L5-A), then there is a positive measure of pairs $s_{L}<s_{H}$ such that $g\left(s_{L}\right)>0$, but $g\left(s_{H}\right)<0$. But then, $k$ can be defined so that $k\left(\cdot ; \theta_{H}\right)$ places all of the weight on high points near $s_{H}$, while $k\left(\cdot ; \theta_{L}\right)$ places all of the weight on the low points near $s_{L}$. This $k$ is log-spm, but $G$ fails SC1.

If $k$ fails (L5-B), then there exist two sets of positive measure, $S_{H}$ and $S_{L}$, such that increasing $\theta$ places more weight on $S_{L}$ relative to $S_{H}$. Then we can construct a $g(s)$ that is negative on $S_{L}$, positive on $S_{H}$, and close to zero everywhere else as illustrated in Figure I.

\section{A. Applications in Investment and Portfolio Theory}

The first set of applications considers investment problems where the agent chooses the probability distribution directly, from a parameterized family of distributions. For example, the agent chooses effort in a principal-agent problem, or makes an investment decision. The exogenous parameter $(\theta)$ describes risk preferences. We seek a MPSC for the conclusion that investment increases when risk preferences change. For this purpose, Lemma 5 provides succinct proofs of some existing results, and further suggests some new ones.

Let $v: S \times \Theta \rightarrow \mathbb{R}$ be an agent's utility function, suppose that $S=[\underline{s}, \bar{s}] \subset \mathbb{R}$, and let $H(\cdot ; x)$ be a probability distribution on $S$, parameterized by $x$, with density $h(\cdot ; x)$ with respect to $\mu$. The agent solves $\max _{x \in B} \int_{S} v(s, \theta) h(s ; x) d \mu(s) .{ }^{30}$ For simplicity, consider the case where $v$ is twice differentiable in $s$, and the derivatives (denoted $v_{s}$ and $v_{s s}$ ) are absolutely continuous. Then,

$$
\begin{aligned}
& \arg \max _{x \in B} \int_{S} v(s, \theta) h(s ; x) d \mu(s)=\arg \max _{x \in B}-\int_{S} v_{s}(s, \theta) H(s ; x) d s \\
& =\arg \max _{x \in B} v_{s}(\bar{s}, \theta) \int_{S} s h(s ; x) d \mu(s)+\int_{S} v_{s s}(s, \theta) \int_{t=\underline{s}}^{s} H(t ; x) d t d s .
\end{aligned}
$$

The following result follows directly from these equations and Lemma $5 .{ }^{31}$

30. This is an example where it is useful to have results that do not rely on concavity of the objective: concavity of the objective in $x$ requires additional assumptions (see Jewitt [1988a] and Athey [1998]), which may or may not be reasonable in a given application.

31. Of these results, only (ii) has received attention in the literature. Dia- 


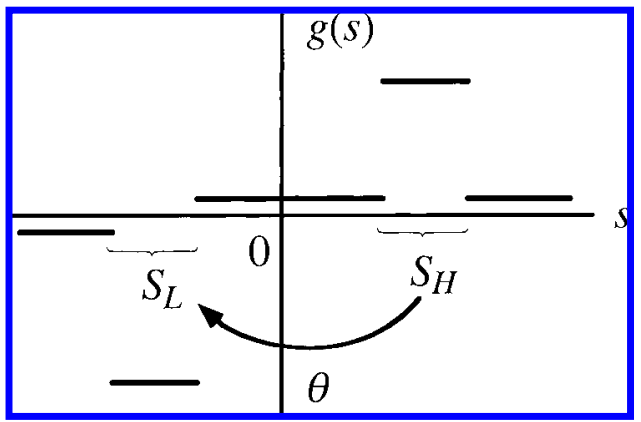

FIGURE I

A Test Function for the Set of Single-Crossing Functions

Proposition 2. Consider the following conclusion: (C) $x *(\theta, B)=$ $\arg \max _{x \in B} \int_{S} v(s, \theta) h(s ; x) d \mu(s)$ is nondecreasing in $\theta$ and $B$. In each of the following, (A) and (B) are a MPSC for (C):

Additional Assumptions:

(i) $v \geq 0$, and $\{s \mid v(s, \theta) \neq 0\}$ is constant in $\theta$.

(ii) $v_{s} \geq 0$, and $\left\{s \mid v_{s}(s, \theta) \neq 0\right\}$ is constant in $\theta$.

(iii) $\int_{s} s f(s ; x) d s$ is constant in $x$, $v_{s s} \leq 0$, and $\left\{s \mid v_{s s}(s, \theta) \neq 0\right\}$ is constant in $\theta$.
(A)

(B)

$v$ is log-spm. a.e.- $\mu$.

$h$ is weak SC2 in $(x ; s)$ a.e. $\mu$.

$v_{s}$ is log-spm. in $(s,-\theta)$ a.e.Lebesgue.

$H$ is weak SC2 in ( $x$; $s)$ a.e.-Lebesgue.

$-v_{s s}(s, \theta)$ is log-spm. in $(s,-\theta)$ a.e.-Lebesgue.

$\int_{-\infty}^{s} H(t ; x) d t$ is WSC2 in $(x ; s)$ a.e.-Lebesgue.

This result provides necessary and sufficient conditions for comparative statics in a range of applications. Consider each of (i)-(iii) in turn. ${ }^{32}$ Case (i) might apply if a principal offers a mechanism to an agent where the allocation that will be received

mond and Stiglitz [1974] established the sufficiency side of the relationship, and many authors have since exploited and further studied the result (such as Jewitt [1987, 1989]). Jewitt [1987] shows that (A) is necessary and sufficient for (C) to hold whenever (B) does.

32. In each of (i)-(iii), the fact that (B) is necessary for the conclusion to hold whenever (A) holds relies crucially on the nonmonotonicity of the relevant function in (A). Thus, while the sufficient conditions and the necessity of (A) in each case are quite general, one should be more careful in drawing conclusions about the necessity of (B). 
is stochastic. ${ }^{33}$ The payoff $u$ is log-spm when higher types have a larger relative return to $s$. When the support of $s$ is fixed, weak SC2 of a probability density is stronger than FOSD but weaker than MLR. In (ii), hypothesis (A) requires that the agent's ArrowPratt risk aversion is nondecreasing in $\theta$. Further, (B) requires $H\left(s ; x_{H}\right)$ crosses $H\left(s ; x_{L}\right)$ at most once, from below, as a function of $s$. Under this assumption, it is possible that increasing $x$ decrease the mean as well as the riskiness of the distribution; that is, it might incorporate a mean-risk trade-off. In case (iii), the agents are restricted to be risk averse. We see that $x$ always increases with an agent's prudence (as defined in subsection III.A) if and only if $\int_{-\infty}^{s} H(t ; x) d t$ satisfies weak SC2 in $(x ; s)$.

The second set of applications considers two classic problems, the portfolio investment problem and the decision problem of a risk-averse firm. We generalize several comparative statics results previously established only for special functional forms.

Consider first the standard portfolio problem, where an agent with initial wealth $w$ invests $x$ in a risky asset with return $s$, and invests the remainder $(w-x)$ in a risk-free asset with return $r$. Thus, the agent's payoff can be written as $u((w-x) r+s x)$. The marginal returns to investment are given by $\int u^{\prime}((w-x) r+$ $s x)(s-r) f(s ; \theta) d \mu(s)$. Notice that $s-r$ satisfies single crossing, and as long as the utility function is nondecreasing, we can apply Lemma 5 to this problem. In this problem the crossing point is fixed at $s=r$ for all choices of $x$ : thus, in applying (2), we could actually weaken the restriction that $f$ is $\log$-spm. In particular, we could assume that the likelihood ratio, $l(s)$, is less than $l(r)$ for $s<$ $r$, and greater than $l(r)$ for $s>r$. On the other hand, if we wish to obtain comparative statics results that hold for all risk-free rates, $r$, then it will be necessary that $l(s)$ is log-spm.

While the portfolio problem has been widely studied, far fewer results have been obtained for more general investment problems, where potentially risk-averse firms invest in a risky project $\pi(x, s)$, or make pricing or quantity decisions under uncertainty about demand. Suppose that an agent's objective is as follows: $\max _{x \in B} \int u(\pi(x, s)) f(s ; \theta) d \mu(s)$, and the solution set is denoted $x *(\theta, B)$. Thus, $\pi$ represents a general return function which depends on the investment amount, $x$, and the state of the world,

33. Such uncertainty might arise if the principal cannot observe the agent's choice perfectly, or if the principal must design an error-prone bureaucratic system to carry out the regulation. 
$s$. Notice that in this problem, the crossing point of $\pi_{x}$ is not the same for all $x$ (as it will be in the portfolio problem).

When this objective function is differentiable, it suffices to check that the marginal returns to $x$, denoted $\int u^{\prime}(\pi(x, s))$ $\pi_{x}(x, s) f(s ; \theta) d \mu(s)$, satisfy SC1. The following result treats this case, as well as cases where investment is a discrete choice, or the agent's risk preferences change with the exogenous parameter. ${ }^{34}$

Proposition 3. Consider the problem $\max _{x \in B} \int u(\pi(x, s)) f(s ; \theta)$ $d \mu(s)$. Assume that $u(y, \theta)$ is increasing and differentiable ${ }^{35}$ in $y$, and $\pi(x, s)$ is nondecreasing in $s$. Then, (A) $\pi(x, s)$ satisfies SC2 in $(x ; s)$ a.e. $\mu$, and (B) $u_{1}(y, \theta) f(s ; \theta)$ is log-spm in $(s, y, \theta)$ a.e. $\mu,{ }^{36}$ are a MPSC for the conclusion $(\mathrm{C}) x^{*}(\theta, B)$ is nondecreasing in $\theta$ and $B$.

Hypothesis (B) is satisfied if (i) $\theta$ decreases the investor's absolute risk aversion, and (ii) $\theta$ generates an MLR shift in $F$. Thus, this result provides a generalization of two basic results in the theory of investment under uncertainty, illustrating that log-supermodularity links the seemingly unrelated conditions on the distribution and the investor's risk aversion.

\section{Incorporating Additional Structure on Primitives}

Many economic primitives have more structure than a singlecrossing property. One particular kind of structure that arises in auction games and investment problems is that the incremental return function $g(s)$ is quasi-concave in $s$. For example, in a portfolio problem with $r=0$, the marginal returns to investment are quasi-concave if the agent is risk averse and the coefficient of relative risk aversion is greater than 1 .

34. This result generalizes the existing investment literature in several ways. This literature typically considers the problem where the objective is differentiable and strictly quasi-concave. Landsberger and Meilijson [1990] show that the MLR is sufficient for comparative statics in the portfolio problem, and Ormiston and Schlee [1993] show that general comparative statics results are preserved by the MLR. A few papers consider comparative statics when $\pi(x, s)=$ $h(x) \cdot s$, as in Sandmo's [1971] classic model of a firm facing demand uncertainty. Milgrom [1994] shows that comparative statics results derived for the portfolio problem also hold for Sandmo's model. Proposition 2 highlights the critical role played by the assumption that $\pi$ satisfies SC2, but not multiplicative separability, extending the existing results to more general models of firm objectives.

35. Differentiability is not essential, but it simplifies the proof.

36 . If $u$ is not everywhere differentiable in its first argument, the corresponding hypothesis can be stated as follows: $\left[u\left(y_{H}, \theta\right)-u\left(y_{L}, \theta\right)\right] \cdot f(s ; \theta)$ is log-supermodular in $\left(y_{L}, y_{H}, \theta, s\right)$ for all $y_{L}<y_{H}$. 
Notice that quasi concavity of $g$ prevents us from constructing the counterexamples of Lemma 5: the test function illustrated in Figure I is clearly not quasi-concave. Theorem 2 can then be extended, as follows.

Theorem 3. For each $\theta \in \Theta$, let $K(\cdot ; \theta)$ be a probability distribution on $S$. Then (C) (MCS') holds for all sets $B$ whenever (A) $u$ satisfies SC2 in $(x ; s)$ a.e.- $\mu .,{ }^{37}$ and for all $x_{H}>x_{L}, u\left(x_{H}, s\right)-$ $u\left(x_{L}, \mathrm{~s}\right)$ is weakly quasi-concave in $s$ a.e.- $\mu$, if and only if $(\mathrm{B}) K$ is log-supermodular.

By Lemma 2 (and recalling the discussion in Section II), log-spm of $K$ (corresponding to the Monotone Probability Ratio Order) is weaker than log-spm of $k$ (corresponding to MLR). To understand the difference between the two orders, observe that the Monotone Probability Ratio Order (MPR) requires that $\theta$ increases the weight on $s$ relative to the aggregate of all states $s^{\prime}$ $<s$, while the MLR requires that $\theta$ increases the weight on $s$ relative to every individual state $s^{\prime}<s .{ }^{38}$ One consequence is that the MPR is more robust to perturbations.

Necessity of (T3-B) can be established using the test functions approach. ${ }^{39}$

\section{Lemma 7 (Test Functions for Single-Crossing/Quasi-Concave} Problems). If we define

$$
\begin{aligned}
\mathscr{g}(\beta) \equiv & \left\{g: \exists a, b>0, \beta>\epsilon, \delta>0, \text { and } s_{0} \text { such that } g(s)=-a\right. \\
& \text { for } s \in\left(-\infty, s_{0}\right], g(s)=b \text { for } s \in\left(s_{0}, s_{0}+\epsilon\right] \\
& \text { and } \left.g(s)=\delta \text { for } s \in\left(s_{0}, \infty\right]\right\},
\end{aligned}
$$

37. We will say that $g(s)$ satisfies SC1 almost everywhere- $\mu$ (a.e. $-\mu$ ) if conditions (a) and (b) of the definition of SC1 hold for almost all (with respect to the product measure on $\mathbb{R}^{2}$ induced by $\left.\mu\right)\left(s_{L}, s_{H}\right)$ pairs in $\mathbb{R}^{2}$ such that $s_{L}<s_{H}$. The definition of SC2 a.e.- $\mu$ is defined analogously.

38. Another way to describe the difference is that the MLR requires that a distribution be ordered by First Order Stochastic Dominance conditional on every two-point set, while the MPR requires a distribution to be ordered by First Order Stochastic Dominance conditional on every interval $(-\infty, s]$ (the latter result is established in the working paper [Athey 1996]).

39. It turns out that quasi concavity of $g$ is not necessary for the comparative statics conclusion. This follows because, as a probability distribution, $K$ is restricted to be monotone. This restriction prevents us from constructing the requisite counterexamples. It can, however, be established that $g$ cannot be decreasing and then increasing at the upper end of the support; more generally, any violations of quasi concavity must not be too severe relative to the magnitude of the function. 


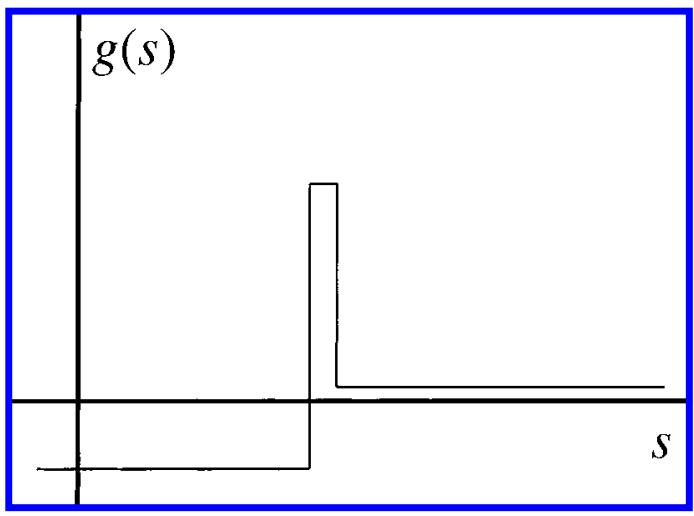

FIGURE II

A Test Function for the Set of Single-Crossing, Quasi-Concave Functions

then Theorem 3 holds if, for any $\beta>0$, (T3-A) is replaced with $\left(T 3-A^{\prime}\right)$ for all $x_{H}>x_{L}, u\left(x_{H}, \cdot\right)-u\left(x_{L}, \cdot\right)=g(\cdot) \in \mathfrak{g}(\beta)$.

Figure II illustrates a test function from Lemma 7 . The expected value of such a function is approximately $b \cdot k\left(s_{0} ; \theta\right)-a \cdot$ $K\left(s_{0} ; \theta\right)$, highlighting the role of monotonicity of $k / K$ in $\theta$ (i.e., log-spm of $K$ ).

Athey [2000] further explores how restrictions on risk preferences affect the comparative statics of portfolio and investment problems; here, we present an example from auction theory.

\section{A. Application: Mineral Rights Auction with Asymmetries and Risk Aversion}

This section studies Milgrom and Weber's [1982] model of a mineral rights auction, generalized to allow for risk-averse, asymmetric bidders whose utility functions are not necessarily differentiable. ${ }^{40}$ We focus on the case of two bidders. Suppose that bidders 1 and 2 observe signals $s_{1}$ and $s_{2}$, respectively, where each agent's utility (written $\left.v_{i}\left(b_{i}, s_{1}, s_{2}\right)\right)$ satisfies

(3) $v_{i}\left(b_{i}, s_{1}, s_{2}\right)$ is nondecreasing in $\left(-b_{i}, s_{1}, s_{2}\right)$ and

$$
\text { supermodular in }\left(b_{i}, s_{j}\right), \quad j=1,2 .
$$

40. As in the pricing game studied in Section II, this is not a game with strategic complementarities between players' bidding functions, so Vives [1990] may not be applied to establish existence of a PSNE. 
The signals have a joint density $h$ with respect to Lebesgue measure, and the conditional distribution of $s_{-i}$ given $s_{i}$ is written as $H_{-i}\left(\cdot \mid s_{i}\right)$, with density $h_{-i}\left(\cdot \mid s_{i}\right)$. Condition (3) holds, for example, if $v_{i}\left(b_{i}, s_{1}, s_{2}\right) \equiv \int \hat{v}_{i}\left(y-b_{i}\right) g\left(y \mid s_{1}, s_{2}\right) d y$, where the "common value" $y$ is affiliated with $s_{1}$ and $s_{2}, g\left(\cdot \mid s_{1}, s_{2}\right)$ is the conditional density of $y$, and $\hat{v}_{i}$ is nondecreasing and concave. ${ }^{41}$

When player 2 uses the bidding function $\beta_{2}(\cdot)$, then the set of best reply bids for player 1 given her signal $\left(s_{1}\right)$ can be written as (assuming ties are broken randomly)

$$
\begin{aligned}
b_{1}^{*}\left(s_{1}\right)=\arg \max _{b_{1} \in B} \int_{s_{2}} & v_{1}\left(b_{1}, s_{1}, s_{2}\right) 1_{b_{1}>\beta_{2}\left(s_{2}\right)}\left(s_{2}\right) h_{2}\left(s_{2} \mid s_{1}\right) d s_{2} \\
& +1 / 2 \int_{s_{2}} v_{1}\left(b_{1}, s_{1}, s_{2}\right) 1_{b_{1}=\beta_{2}\left(s_{2}\right)}\left(s_{2}\right) h_{2}\left(s_{2} \mid s_{1}\right) d s_{2} .
\end{aligned}
$$

When bidder 2 plays a nondecreasing strategy, (3) implies that bidder 1's payoff function given a realization of $s_{2}$ satisfies weak SC2 in $\left(b_{1} ; s_{2}\right)$. Consider first the case where player 2's strategy is strictly increasing. The returns to increasing the bid from $b_{L}$ to $b_{H}$ are strictly negative for low values of $s_{2}$, when the opponent bids less than $b_{L}$; the returns are increasing in $s_{2}$ on the region where raising the bid causes the player to win, where she would have lost with $b_{L}$; and the effect is zero for $s_{2}$ so high that even $b_{H}$ does not win. Thus, the incremental returns to the bid are quasi-concave as well as weak single crossing. When ties are permitted, the single-crossing property remains, but quasi concavity fails.

Observe further that the payoff function depends directly on $s_{1}$ as well. By assumption, the returns to $b_{1}$ are increasing in $s_{1}$. This yields (using Extension (i) to Lemma 5, and Theorem 3).

Proposition 4. Consider the two-bidder mineral rights model, where the utility function satisfies (3) above, and the support of the random variables is a product set. (i) Suppose that bidder 2 uses a strategy $\beta_{2}(\cdot)$ which is nondecreasing in $s_{2}$. Then if the types are affiliated, $b_{1}^{*}(\cdot)$ is nondecreasing in $s_{1}$. (ii) Suppose that bidder 2 uses a strategy $\beta_{2}(\cdot)$ which is

41. To see why (3) holds, note that $s_{i}$ and $s_{j}$ each induce a first-order stochastic dominance shift on $F$, and $\hat{u}_{i}$ is supermodular in $\left(b_{i}, v\right)$. Supermodularity of the expectation in $\left(b_{i}, s_{i}\right)$ and $\left(b_{i}, s_{j}\right)$ follows (see Athey [1998]). 
strictly increasing in $s_{2}$. Then if $H_{2}\left(s_{2} \mid s_{1}\right)$ is log-spm in $\left(s_{1}, s_{2}\right)$, $b_{1}^{*}(\cdot)$ is nondecreasing in $s_{1}$.

Athey [2001] applies this result to show that in auction games such as the first price auction described above, a PSNE exists. ${ }^{42}$

\section{Single Crossing of Indifference Curves}

The Spence-Mirrlees single-crossing property (SM) is central to the analysis of monotonicity in standard signaling and screening games, as well as many other mechanism design problems. For an arbitrary differentiable function $h: \mathbb{R}^{3} \rightarrow \mathbb{R}$ that satisfies $(\partial / \partial y) h(x, y, t) \neq 0,(\mathrm{SM})$ is defined as follows:

$$
\frac{\partial}{\partial x} h(x, y, t) /\left|\frac{\partial}{\partial y} h(x, y, t)\right| \text { is nondecreasing in } t .
$$

When the $(x, y)$ indifference curves are well defined, $(\mathrm{SM})$ is equivalent to the requirement that the indifference curves cross at most once as a function of $t$. We will make use of the following assumption which guarantees that the $(x, y)$-indifference curves are well-behaved: ${ }^{43}$

(WB) $h$ is differentiable in $(x, y) ; \frac{\partial}{\partial y} h(x, y, t) \neq 0$;

$$
\text { the }(x, y) \text {-indifference curves are closed curves. }
$$

The following result characterizes (SM) for objective functions of the form, $V(x, y, \theta) \equiv \int v(x, y, s) f(s ; \theta) d \mu(s)$.

Lemma 8. Let $v: \mathbb{R}^{3} \rightarrow \mathbb{R}$ and $f: \mathbb{R}^{2} \rightarrow \mathbb{R}_{+}$, and suppose that $v$ and $V$ satisfy (WB). Then (A) $v(x, y, s)$ satisfies (SM) a.e. $-\mu$, and (B) $f$ is $\log$-spm in $(s ; \theta)$ a.e.- $\mu$, are a MPSC for $(\mathrm{C}) V(x, y, \theta)$ satisfies (SM).

42. What happens when we try to extend this model to $I \geq 2$ bidders? If the bidders face a symmetric distribution, and all opponents use the same symmetric bidding function, then only the maximum signal of all of the opponents will be relevant to bidder 1 . Define $s_{M}=\max \left(s_{2}, \ldots, s_{1}\right)$. Milgrom and Weber [1982] show that $\left(s_{1}, s_{M}\right)$ are affiliated when the distribution is exchangeable. Further, if the opponents are using the same strategies, whichever opponent has the highest signal will necessarily have the highest bid. Then we can apply Proposition 4 to this problem exactly as if there were only two bidders. Unfortunately, this approach does not extend directly to $n$-bidder, asymmetric auctions with common value elements. Under asymmetric distributions (or if players use asymmetric strategies), affiliation of the signals is not sufficient to guarantee that the signal of the highest bidder is affiliated with a given player's signal, nor is it sufficient to guarantee log-supermodularity of the conditional distribution.

43. It is also possible to generalize SM to the case where $h$ is not differentiable, but we maintain (WB) for simplicity. 
Theorem 4 (Comparative Statics and the Spence-Mirrlees SCP). Lemma 8 also holds if (L8-C) is replaced with $(\mathrm{C}) x^{*}(\theta, B)=$ $\arg \max _{x \in B} V(x, b(x), \theta)$ is nondecreasing in $\theta$ and $B$ for all $b$ : $\mathbb{R} \rightarrow \mathbb{R}$.

Sufficiency in Lemma 8 can be shown using Lemma 2. Let: $h_{1}(s)=\left|v_{y}(x, y, s)\right| f\left(s ; \theta_{L}\right), h_{2}(s)=v_{x}(x, y, s) f\left(s ; \theta_{H}\right), h_{3}(s)=v_{x}(x, y, s) f(s ;$ $\left.\theta_{H}\right), h_{4}(s)=\left|v_{y}(x, y, s)\right| f\left(s ; \theta_{L}\right)$, and note that (T5-A) and (T5-B) imply that for all $S_{H}>S_{L}, \theta_{H}>\theta_{L}$

$$
\frac{v_{x}\left(x, y, s_{L}\right)}{\left|v_{y}\left(x, y, s_{L}\right)\right|} \frac{f\left(s_{L} ; \theta_{H}\right)}{f\left(s_{L} ; \theta_{L}\right)} \leq \frac{v_{x}\left(x, y, s_{H}\right)}{\left|v_{y}\left(x, y, s_{H}\right)\right|} \frac{f\left(s_{H} ; \theta_{H}\right)}{f\left(s_{H} ; \theta_{L}\right)} \text {. }
$$

This in turn implies by Lemma 2 that

$$
\frac{\partial}{\partial x} V\left(x, y, \theta_{L}\right) /\left|\frac{\partial}{\partial y} V\left(x, y, \theta_{L}\right)\right| \leq \frac{\partial}{\partial x} V\left(x, y, \theta_{H}\right) /\left|\frac{\partial}{\partial y} V\left(x, y, \theta_{H}\right)\right| .
$$

\section{A. Applications to Signaling Games and Savings Problems}

Theorem 4 can be applied to an education signaling model, where $x$ represents a worker's choice of education, $y$ is monetary income, and $\theta$ is a noisy signal of the worker's ability, $s$ (for example, the workers' experience in previous schooling). If the worker's preferences $u(x, y, s)$ satisfy (SM) and higher signals increase the likelihood of high ability, the worker's education choice will be nondecreasing in the signal $\theta$ for any wage function $w(x)$.

In another example, consider a consumption-savings problem. Let $x$ denote savings and $b(\cdot)$ the value function of savings. The agent has an endowment $(\theta)$ of a risky asset $(s)$. The probability distribution over asset returns is given by $F(\cdot ; \theta)$. The agent's utility given a realization of $s$ is $u(z+s-x, b(x))$, which is assumed to be nondecreasing. The agent solves $\max _{x} \in[0, z]$ $\int u(z+s-x, b(x)) d F(s ; \theta)$. The following two conditions are a minimal pair of sufficient conditions for the conclusion that savings increases in $\theta$ : (A) the marginal rate of substitution of current for future utility, $u_{1} / u_{2}$, is nondecreasing in $s$, and (B) $F$ satisfies MLR.

\section{Conclusions}

This paper studies conditions for comparative statics predictions in stochastic problems, and shows how they apply to economic problems. The main theorems are summarized in Table I. 


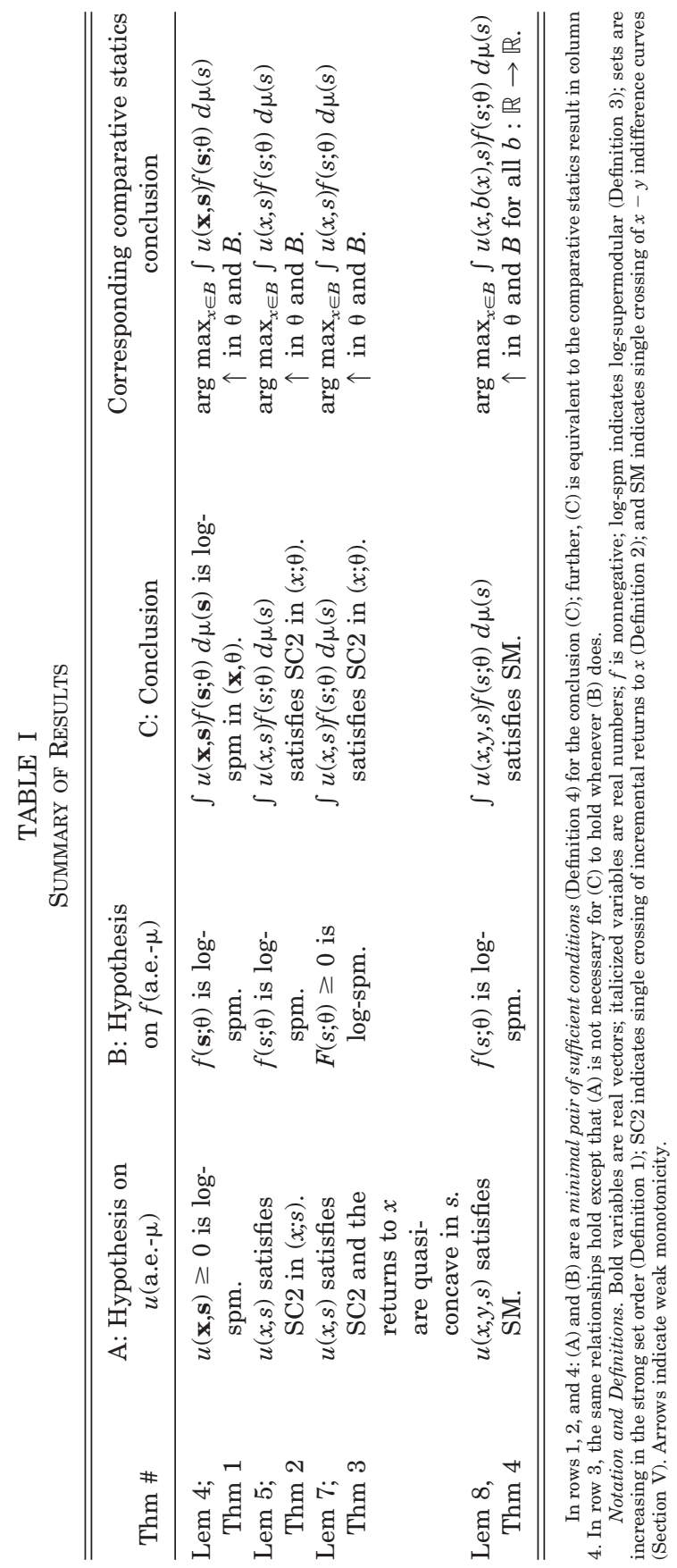


The properties of primitives considered in this paper, the various single-crossing properties and log-supermodularity, are each necessary and sufficient for comparative statics in an appropriately defined class of problems. Several variations of these results are analyzed, each of which exploits additional structure that can arise in economic problems. Because the properties studied in this paper, and the corresponding comparative statics predictions, do not rely on differentiability or concavity, the results from this paper can be applied in a wider variety of economic contexts than similar results from the existing literature.

This paper builds on results from the statistics literature to establish sufficient conditions for comparative statics. We further provide new results about necessity, while highlighting the limitations of these results. Table I summarizes the trade-offs that must occur between weakening and strengthening assumptions about various components of economic models. Together with Athey [1998]'s analysis of stochastic supermodularity, concavity, and other differential properties, the results in this paper can be used to identify which assumptions are the appropriate ones to guarantee robust monotone comparative statics predictions in a wide variety of stochastic problems in economics.

\section{APPENDIX}

Definition A1. For each $\theta$, let $F(\cdot ; \theta): S \rightarrow \mathbb{R}_{+}$be nondecreasing and right-continuous. For all $\theta_{H}>\theta_{L}$, let $C^{L H}(\cdot)=1 / 2\left[F\left(\cdot ; \theta_{H}\right)\right.$ $\left.+F\left(\cdot ; \theta_{L}\right)\right]$, and define $f^{L H}: S \times\left\{\theta_{L}, \theta_{H}\right\} \rightarrow \mathbb{R}_{+}$so that for all $(s, \theta)$, $F(s ; \theta)=\int_{-\infty}^{s} f^{L H}(t ; \theta) d C^{L H}(t)$. The parameter $\theta$ indexes $F(\cdot ; \theta)$ according to the Monotone Likelihood Ratio Order (MLR) if, for all $\theta_{H}>\theta_{L}, f^{L H}$ is log-spm a.e.- $\mu .{ }^{44}$

Proof of Lemma 1. If $U$ is log-spm in $(\mathbf{x}, \theta)$, then it must be quasi-spm, which Milgrom and Shannon [1994] show implies the comparative statics conclusion. Now suppose that $U$ fails to be $\log$-spm in $(\mathbf{x}, \theta)$ for some $u$. For simplicity, consider the case where $n=2$, and start with the case where $U>0$. Then, there exists an $x_{1 H}>x_{1 L}, x_{2 H} \geq x_{2 L}$ and $\theta_{H} \geq \theta_{L}$, with one of the weak

44. This definition is equivalent to the standard one, except that the definition is more inclusive, in that absolute continuity of $F\left(\cdot ; \theta_{H}\right)$ with respect to $F\left(\cdot ; \theta_{L}\right)$ on the intersection of their supports is a consequence of the definition, not prerequisite for comparability. 
inequalities holding strictly, such that $U\left(x_{1 H}, x_{2 H}, \theta_{H}\right) / U\left(x_{1 L}, x_{2 H}, \theta_{H}\right)$ $<U\left(x_{1 H}, x_{2 L}, \theta_{L}\right) / U\left(x_{1 L}, x_{2 L}, \theta_{L}\right)$. Let $\gamma=U\left(x_{1 L}, x_{2 L}, \theta_{L}\right) /$ $U\left(x_{1 H}, x_{2 L}, \theta_{L}\right)>0$. Then, define $b: X_{1} \rightarrow \mathbb{R}$ by $b\left(x_{1}\right)=1$ if $x_{1} \neq x_{1 H}$, and $b\left(x_{1 H}\right)=\gamma$. Since log-spm is preserved by multiplication, $v(\mathbf{x}, \mathbf{s}) \equiv u(\mathbf{x}, \mathbf{s}) \cdot b\left(x_{1}\right)$ is log-spm in $(\mathbf{x}, \mathbf{s})$. Define $V(\mathbf{x}, \theta) \equiv$ $\int v(\mathbf{x}, \mathbf{s}) f(\mathbf{s} ; \theta) d \mu(\mathbf{s})$. But then, $V\left(x_{1 H}, x_{2 H}, \theta_{H}\right) / V\left(x_{1 L}, x_{2 H}, \theta_{H}\right)=$ $\gamma U\left(x_{1 H}, x_{2 H}, \theta_{H}\right) / U\left(x_{1 L}, x_{2 H}, \theta_{H}\right)<1=\gamma U\left(x_{1 H}, x_{2 L}, \theta_{L}\right) / U\left(x_{1 L}, x_{2 L}, \theta_{L}\right)=$ $V\left(x_{1 H}, x_{2 L}, \theta_{L}\right) / V\left(x_{1 L}, x_{2 L}, \theta_{L}\right)$. Thus, $V\left(x_{1 H}, x_{2 L}, \theta_{L}\right)=V\left(x_{1 L}, x_{2 L}, \theta_{L}\right)$ while $V\left(x_{1 H}, x_{2 H}, \theta_{H}\right)<V\left(x_{1 L}, x_{2 H}, \theta_{H}\right)$, violating the requirement that (a) $V$ is quasi-spm in $\mathbf{x}$ and (b) $V$ satisfies SC2 in $(\mathbf{x} ; \theta)$.

Now, return to the case where we might have $U(\mathbf{x}, \theta)=0$ for some $(\mathbf{x}, \theta)$. Note that since $U \geq 0$, log-spm of $U$ can fail in the example above only if $U\left(x_{1 H}, x_{2 L}, \theta_{L}\right)>0$ and $U\left(x_{1 L}, x_{2 H}, \theta_{H}\right)>0$. If, in addition, $U\left(x_{1 L}, x_{2 L}, \theta_{L}\right)>0$, the argument above is unchanged. Now suppose that $U\left(x_{1 L}, x_{2 L}, \theta_{L}\right)=0$. If $U\left(x_{1 H}, x_{2 H}, \theta_{H}\right)=0$, then quasi supermodularity fails as well; if $U\left(x_{1 H}, x_{2 H}, \theta_{H}\right)>0$, then define $v$ as above except with $\gamma=U\left(x_{1 L}, x_{2 H}, \theta_{H}\right) / U\left(x_{1 H}, x_{2 H}, \theta_{H}\right)$. Then, we have $V\left(x_{1 H}, x_{2 L}, \theta_{L}\right)>V\left(x_{1 L}, x_{2 L}, \theta_{L}\right)=0$ while $V\left(x_{1 H}, x_{2 H}, \theta_{H}\right)=V\left(x_{1 L}, x_{2 H}, \theta_{H}\right)$, another violation of Milgrom and Shannon's [1994] necessary and sufficient conditions for comparative statics.

Proof of Lemma 4. Sufficiency follows from Lemma 2. Necessity is treated in two main cases, with other cases following in a similar manner:

(1) $n, m=1$ : Define $v(A \mid \theta) \equiv \int_{A} f(s ; \theta) d \mu(s)$. Pick any two intervals of length $\epsilon, S_{H}(\epsilon)$ and $S_{L}(\epsilon)$, such that $S_{H}(\epsilon) \geq S_{L}(\epsilon)$ and $S_{H}(\epsilon) \cap S_{L}(\epsilon)=\varnothing$. Define $u:\left\{x_{L}, x_{H}\right\} \times \mathbf{S} \rightarrow \mathbb{R}$ by $u\left(x_{L}, s\right) \equiv 1_{S_{L}(\epsilon)}(s)$, and let $u\left(x_{H}, s\right) \equiv 1_{S_{H}(\epsilon)}(s)$. Then $\int u\left(x_{L}, s\right) f(s, \theta) d \mu(s)=v\left(S_{L} \mid \theta\right)$ and $\int u\left(x_{H}, s\right) f(s ; \theta) d \mu(s)=v\left(S_{H} \mid \theta\right)$. Since $u$ is log-spm, then $\int u(x, s) f(s ; \theta)$ $d \mu(s)$ must be log-spm by (C), i.e., $v\left(S_{H}, \theta_{H}\right) v\left(S_{L}, \theta_{L}\right) \geq$ $v\left(S_{H}, \theta_{L}\right) v\left(S_{L}, \theta_{H}\right)$. Taking the limit as $\epsilon \rightarrow 0$, standard limiting arguments (e.g., Martingale convergence theorem) imply that $f$ must be log-spm in $(s, \theta)$ almost everywhere- $\mu$.

(2) $n, m \geq 2$ : Define $v(A \mid \theta)=\int_{A} f(\mathbf{s} ; \theta) d \mu(\mathbf{s})$. We partition $\mathbb{R}^{\mathbf{m}}$ into $m$-cubes with element $i$ given by $\left[i_{1}-1 / 2^{t}, i_{1}+1 / 2^{t}\right] \times \cdots \times$ $\left[i_{m}-1 / 2^{t}, i_{m}+1 / 2^{t}\right]$, and let $\mathbf{Q}^{t}(\mathbf{s})$ be the unique cube containing s. Consider a $t$, and take any $\mathbf{a}, \mathbf{b} \in \mathbb{R}^{\mathrm{m}}$. Further, let $C=\{\mathbf{y}, \mathbf{z}, \mathbf{y} \vee$ $\mathbf{z}, \mathbf{y} \wedge \mathbf{z}\}$, where each element of $C$ is distinct. Define $u(\mathbf{x}, \mathbf{s})$ on $C$ as follows: $u(\mathbf{y}, \mathbf{s})=1_{Q^{t}(\mathbf{a})}(\mathbf{s}), u(\mathbf{z}, \mathbf{s})=1_{Q^{t}(\mathbf{b})}(\mathbf{s}), u(\mathbf{y} \vee \mathbf{z}, \mathbf{s})=1_{Q^{t}(\mathbf{a} \vee \mathbf{b})}(\mathbf{s})$, and $u(\mathbf{y} \wedge \mathbf{z}, \mathbf{s})=1_{Q^{t}(\mathbf{a} \wedge \mathbf{b})}(\mathbf{s})$. Let $u(\mathbf{x}, \mathbf{s})=0$ for $\mathbf{x} \notin C$. It is 
straightforward to verify that $u$ is log-spm. If $\int u(\mathbf{x}, \mathbf{s}) f(\mathbf{s} ; \theta) d \mu(\mathbf{s})$ is log-spm, it follows that $v\left(Q^{t}(\mathbf{a} \vee \mathbf{b}) \mid \theta \vee \theta^{\prime}\right) \cdot v\left(Q^{t}(\mathbf{a} \wedge \mathbf{b}) \mid \theta \vee \theta^{\prime}\right) \geq$ $v\left(\boldsymbol{Q}^{t}(\mathbf{a}) \mid \theta\right) \cdot v\left(\boldsymbol{Q}^{t}(\mathbf{b}) \mid \theta^{\prime}\right)$ for all $\theta, \theta^{\prime}$. Since this must hold for all $t$ and for all $\mathbf{a}, \mathbf{b}$, we can use the Martingale convergence theorem to conclude that $f\left(\mathbf{a} \vee \mathbf{b} ; \theta \vee \theta^{\prime}\right) \cdot f\left(\mathbf{a} \wedge \mathbf{b} ; \theta \wedge \theta^{\prime}\right) \geq f(\mathbf{a} ; \theta) \cdot f\left(\mathbf{b} ; \theta^{\prime}\right)$ for $\mu$-almost all $\mathbf{a}, \mathbf{b}$ (recalling that $\mu$ is a product measure).

Proof of Proposition 1. Sufficiency follows from Lemma 2 and Milgrom and Shannon [1994]. Following the proof of Theorem 1, it is possible to show that $U_{i}\left(x_{i}, t_{i}\right)$ is log-supermodular for all $u_{i}\left(x_{i}, \mathbf{t}\right)$ log-supermodular, only if, for all $\mathbf{t}_{-i}^{H}>\mathbf{t}_{-i}^{L}$ and all $t_{i}^{H}>t_{i}^{L}$, $h_{i}\left(\mathbf{t}_{-i}^{H} \mid t_{i}^{H}\right) h_{i}\left(\mathrm{t}_{-i}^{L} \mid t_{i}^{L}\right) \geq h_{i}\left(\mathbf{t}_{-i}^{L} \mid t_{i}^{H}\right) h_{i}\left(\mathbf{t}_{-i}^{H} \mid t_{i}^{L}\right)$. But, since for a positive function, log-spm can be checked pairwise, this condition holds for all $i$ if and only if $h$ is log-spm. Apply Lemma 1 .

Lemma A1. Let $K(s ; \theta) \equiv \int_{-\infty}^{s} k(t ; \theta) d \mu(t) . G(\theta)=\int g( \pm ; \theta) k(s ; \theta) d \mu(s)$ satisfies $\mathrm{SC} 1$ in $\theta$ under the following sufficient conditions: (i)(a) For each $\theta$, $g$ satisfies WSC1 in $s$ a.e.- $\mu$; for $\mu$-almost all $s, g$ is nondecreasing in $\theta$. (i)(b) $k$ is log-spm in $(s, \theta)$ a.e. $-\mu$. (i)(c) Either $g$ satisfies $\mathrm{SC} 1$ in $s$ a.e. $-\mu$, or else supp $[K(\cdot ; \theta)]$ is constant in $\theta$.

Proof of Lemma A1. Pick $\theta_{H}>\theta_{L}$. Suppose that $\int g\left(s ; \theta_{L}\right) k(s$; $\left.\theta_{L}\right) d \mu(s) \geq(>) 0$. Choose $s_{0}$ so that $g\left(s, \theta_{L}\right) \geq 0$ for $\mu$-almost all $s>$ $s_{0}$ and $g\left(s, \theta_{L}\right) \leq 0$ for $\mu$-almost all $s \leq s_{0}\left(s_{0}\right.$ exists by the definition of WSC1). Let $\hat{s}_{0}=\min \left\{s \geq s_{0}\right.$ and $\left.s \in \operatorname{supp}\left[K\left(\cdot ; \theta_{L}\right)\right]\right\}$. First, a fact that we will use repeatedly: if $k$ is $\log$-spm, then $\operatorname{supp}[K(\cdot ; \theta)]$ is increasing in the strong set order. Now, observe that if $\hat{s}_{0} \notin$ supp $\left[K\left(\cdot ; \theta_{H}\right)\right]$, then $\operatorname{supp}\left[K\left(\cdot ; \theta_{H}\right)\right]>\hat{s}_{0}$ since $\operatorname{supp}[K(\cdot ; \theta)]$ is nondecreasing in the strong set order. This in turn implies that, for all $s$ in $\operatorname{supp}\left[K\left(\cdot ; \theta_{H}\right)\right], g\left(s, \theta_{H}\right) \geq g\left(s, \theta_{L}\right) \geq 0$, which implies that $G\left(\theta_{H}\right) \geq 0$. Further, if $G\left(\theta_{L}\right)>0$, then we can conclude that $G\left(\theta_{H}\right)>0$ by (i)(c). Second, observe that the case where supp $\left[K\left(\cdot ; \theta_{H}\right)\right] \leq \hat{s}_{0}$ is degenerate, since this would imply by the strong set order that supp $\left[K\left(\cdot ; \theta_{L}\right)\right] \leq \hat{s}_{0}$ as well. But then our hypothesis that $G\left(\theta_{L}\right)$ is nonnegative would imply that $g\left(s, \theta_{L}\right)=0$ a.e. $\mu$ on supp $\left[K\left(\cdot ; \theta_{L}\right)\right]$. Since supp $[K(\cdot ; \theta)]$ is nondecreasing in the strong set order, this in turn implies that $G\left(\theta_{H}\right)=0$ by (i)(c).

So, we consider the third case where $\hat{s}_{0} \in \operatorname{supp}\left[K\left(\cdot ; \theta_{H}\right)\right]$, but there exist $s^{\prime}, s^{\prime \prime} \in \operatorname{supp}\left[K\left(\cdot ; \theta_{H}\right)\right]$ such that $s^{\prime}<\hat{s}_{0}<s^{\prime \prime}$. Notice that, by the strong set order, supp $\left[K\left(\cdot ; \theta_{H}\right)\right]=\operatorname{supp}$ $\left[K\left(\cdot ; \theta_{L}\right)\right]$ on an interval surrounding $\hat{s}_{0}$ unless $\hat{s}_{0} \geq \operatorname{supp}$ $\left[K\left(\cdot ; \theta_{L}\right)\right]$, a trivial case. It further implies that $g\left(s, \theta_{L}\right) \leq 0$ for 
all $s \in \operatorname{supp}\left[K\left(\cdot ; \theta_{L}\right)\right] \backslash \operatorname{supp}\left[K\left(\cdot ; \theta_{H}\right)\right]$ (because everything in the set lies below supp $\left[K\left(\cdot ; \theta_{H}\right)\right]$, which contains $\left.\hat{s}_{0}\right)$. By the same reasoning, $g\left(s, \theta_{L}\right) \geq 0$ on supp $\left[K\left(\cdot ; \theta_{H}\right)\right] \backslash \operatorname{supp}\left[K\left(\cdot ; \theta_{L}\right)\right]$.

Now, define a modified likelihood ratio $\hat{l}(s)$, as follows: $\hat{l}(s)=0$ for $s \notin \operatorname{supp}\left[K\left(\cdot ; \theta_{L}\right)\right], \hat{l}(s)=k\left(s ; \theta_{H}\right) / k\left(s ; \theta_{L}\right)$ for $s \in \operatorname{supp}$ $\left[K\left(\cdot ; \theta_{L}\right)\right]$ and $k\left(s ; \theta_{L}\right)>0$, and then extend the function so that $\hat{l}(s)=\max \left(\lim _{s^{\prime} \downarrow s} \hat{l}\left(s^{\prime}\right), \lim _{s^{\prime} \uparrow s} \hat{l}\left(s^{\prime}\right)\right)$ for $s \in \operatorname{supp}\left[K\left(\cdot ; \theta_{L}\right)\right]$ and $k\left(s ; \theta_{L}\right)=0$ (recalling that the likelihood ratio can be assumed to be nondecreasing in $s$ on $\operatorname{supp}\left[K\left(\cdot ; \theta_{L}\right)\right]$ without loss of generality by $(\mathrm{i})(\mathrm{b}))$. Thus, we know $\hat{l}\left(\hat{s}_{0}\right)>0$, since $\hat{s}_{0} \in \operatorname{supp}$ $\left[K\left(\cdot ; \theta_{L}\right)\right]$ and since $\operatorname{supp}\left[K\left(\cdot ; \theta_{H}\right)\right]=\operatorname{supp}\left[K\left(\cdot ; \theta_{L}\right)\right]$ on an open interval surrounding $\hat{s}_{0}$. We use this to establish

$$
\begin{aligned}
\int g\left(s ; \theta_{H}\right) k\left(s ; \theta_{H}\right) d \mu(s) \geq & \int\left(s ; \theta_{L}\right) k\left(s ; \theta_{H}\right) d \mu(s) \\
\geq & \int\left(s ; \theta_{L}\right) \hat{l}(s) k\left(s ; \theta_{L}\right) d \mu(s) \\
\geq & -l\left(\hat{s}_{0}\right) \int_{-\infty}^{\hat{s}_{0}}\left|g\left(s ; \theta_{L}\right)\right| k\left(s ; \theta_{L}\right) d \mu(s) \\
& +l\left(\hat{s}_{0}\right) \int_{\hat{s}_{0}}^{\infty} g\left(s ; \theta_{L}\right) k\left(s ; \theta_{L}\right) d \mu(s) \\
= & l\left(\hat{s}_{0}\right) \int g\left(s ; \theta_{L}\right) k\left(s ; \theta_{L}\right) d \mu(s) .
\end{aligned}
$$

The first inequality follows by the fact that $g\left(s, \theta_{H}\right) \geq g\left(s, \theta_{L}\right)$. The second inequality follows by the definition of $\hat{l}(s)$ and since $g\left(s, \theta_{L}\right) \geq 0$ on $\operatorname{supp}\left[K\left(\cdot ; \theta_{H}\right)\right] \backslash \operatorname{supp}\left[K\left(\cdot ; \theta_{L}\right)\right]$. The third inequality is true by WSC1 of $g$ and because $\hat{l}(s)$ is nondecreasing a.e.- $\mu$ on supp $\left[K\left(\cdot ; \theta_{L}\right)\right]$ since $k$ is log-spm. The last equality is definitional. Thus, since $\hat{l}(s)>0, G\left(\theta_{L}\right) \geq(>) 0$ implies $G\left(\theta_{H}\right) \geq(>) 0$.

Lemma A2. If $\int g(s) d K(s ; \theta)$ satisfies SC1 whenever $g$ satisfies $\mathrm{SC} 1$, then (i) supp $[K(\cdot ; \theta)]$ is nondecreasing in the strong set order, and (ii) for all $\theta_{H}>\theta_{L}, K\left(\cdot ; \theta_{H}\right)$ is absolutely 
continuous with respect to $K\left(\cdot ; \theta_{L}\right)$ on $\left(\inf _{s} \operatorname{supp}\left[K\left(\cdot ; \theta_{H}\right)\right]\right.$, $\left.\sup _{s} \operatorname{supp}\left[K\left(\cdot ; \theta_{L}\right)\right]\right)$.

Proof of Lemma A2. Pick $\theta_{H}>\theta_{L}$. Define measures $v_{L}$ and $v_{H}$ as follows. $v_{L}(A)=\int_{A} d K\left(s ; \theta_{L}\right)$ and $v_{H}(A)=\int_{A} d K\left(s ; \theta_{H}\right)$. Define $a=\inf \left\{s \mid s \in \operatorname{supp}\left[K\left(\cdot ; \theta_{H}\right)\right]\right\}$ and $b=\sup \left\{s \mid s \in \operatorname{supp}\left[K\left(\cdot ; \theta_{L}\right)\right]\right\}$. Let $C\left(\cdot ; \theta_{H}, \theta_{L}\right)=1 / 2\left[K\left(\cdot ; \theta_{L}\right)+K\left(\cdot ; \theta_{H}\right)\right]$, and define $h(s ; \theta) \equiv$ $d K\left(s ; \theta_{H}\right) / d C\left(s ; \theta_{H}, \theta_{L}\right)$. Let $D \equiv \operatorname{supp}\left[K\left(\cdot ; \theta_{L}\right)\right] \cup \operatorname{supp}\left[K\left(\cdot ; \theta_{H}\right)\right]$. Since the behavior of $h(s ; \theta)$ outside of $D$ will not matter, we will restrict attention to $D$. The proof proceeds in several steps.

Part (a). If $a \geq b$, then the conclusions hold automatically. Throughout the rest of the proof, we treat the case where $a<b$.

Part (b). For any $S \equiv\left(s_{L}, s_{H}\right] \subset[a, b], v_{L}(S)>0$ implies that $v_{H}(S)>0$. Proof. Suppose that $v_{L}(S)>0$ and $v_{H}(S)=0$. Note that $0<K\left(s_{L} ; \theta_{H}\right)$ since $a<s_{L}$. If supp $\left[K\left(\cdot ; \theta_{H}\right)\right] \leq s_{L}$, then define $g$ as follows: $g(s)=-1$ for $s \in\left(-\infty, s_{L}\right)$, while $g(s)=K\left(s_{L} ; \theta_{L}\right) / v_{L}\left(\left[s_{L}, \infty\right)\right)$ for $s \in\left[s_{L}, \infty\right)$. Otherwise, define $g$ as follows: $g(s)=-1$ for $s \in$ $\left(-\infty, s_{L}\right), g(s)=K\left(s_{L} ; \theta_{L}\right) / v_{L}(S)$ for $s \in S$, and $g(s)=.9 \cdot K\left(s_{L} ; \theta_{H}\right) /$ $v_{H}\left(\left[s_{H}, \infty\right)\right)$ for $s \in\left[s_{H}, \infty\right)$. Now, it is straightforward to verify that $\mathrm{SC} 1$ is violated for this $g$.

Part (c). For any $S \equiv\left(s_{L}, s_{H}\right] \subset[a, b], v_{H}(S)>0$ implies that $v_{L}(S)>0$. Proof. Suppose that $v_{H}(S)>0$ and $v_{L}(S)=0$. If $K\left(s_{L} ; \theta_{L}\right)=0$, then define $g$ as follows: $g(s)=-1$ for $s \in\left(-\infty, s_{H}\right)$, while $g(s)=K\left(s_{H} ; \theta_{H}\right) /\left(2 v_{H}\left(\left[s_{H}, \infty\right)\right)\right.$ for $s \in\left[s_{H}, \infty\right)$. Otherwise, define $g$ as follows. $g(s)=-v_{L}\left(\left[s_{H}, \infty\right)\right) / K\left(s_{L} ; \theta_{L}\right)$ for $s \in\left(-\infty, s_{L}\right), g(s)=$ $-v_{H}\left(\left[s_{H}, \infty\right)\right) / v_{H}(S)$ for $s \in S$, and $g(s)=1$ for $s \in\left[s_{H}, \infty\right)$. Now, it is straightforward to verify that SC1 is violated for this $g$.

Part $(d)$. supp $\left[K\left(\cdot ; \theta_{H}\right)\right] \geq \operatorname{supp}\left[K\left(\cdot ; \theta_{L}\right)\right]$ in the strong set order. Proof. Parts (c) and (d) imply that $v_{H}$ is absolutely continuous with respect to $v_{L}$ on $[a, b]$, and vice versa. It now suffices to show that if $s^{\prime} \in \operatorname{supp}\left[K\left(\cdot ; \theta_{H}\right)\right]$ and $s^{\prime \prime} \in \operatorname{supp}\left[K\left(\cdot ; \theta_{L}\right)\right]$, and $s^{\prime \prime}$ $>s^{\prime}$, then $s^{\prime}, s^{\prime \prime} \in \operatorname{supp}\left[K\left(\cdot ; \theta_{L}\right)\right] \cap \operatorname{supp}\left[K\left(\cdot ; \theta_{H}\right)\right]$. Since $s \notin \operatorname{supp}$ $\left[K\left(\cdot ; \theta_{L}\right)\right]$ for all $s>b$, we may restrict attention to $s^{\prime \prime} \leq b$. Likewise we may restrict attention to $s^{\prime}>a$. But, if (as we argued in part (b)) $v_{H}$ is absolutely continuous with respect to $v_{L}$ and vice versa on $[a, b]$, then $\operatorname{supp}\left[K\left(\cdot ; \theta_{H}\right)\right]=\operatorname{supp}\left[K\left(\cdot ; \theta_{L}\right)\right]$ on $[a, b]$, and we are done.

Proof of Lemmas 5 and 6. Sufficiency follows from Lemma A1. Necessity will follow by constructing counterexamples from the relevant sets. Necessity of (L5-A) follows by observing that for 
any $s_{L}<s_{H}$, if we let $S_{L}(\epsilon)=\left(s_{L}-\epsilon, s_{L}\right]$ and $S_{H}(\epsilon)=\left(s_{H}-\epsilon, s_{H}\right]$, the function $k$ defined by $k\left(s ; \theta_{L}\right) \equiv 1_{S_{L}(\epsilon)}(s), k\left(s ; \theta_{H}\right) \equiv 1_{S_{H}(\epsilon)}(s)$ is log-spm. Thus, (L5-C) will imply that $g$ is SC1 a.e.- $\mu$. Necessity of (L5-B): pick $\theta_{H}>\theta_{L}$, and define $v_{L}, v_{H}, a, b, h$, and $D$ as in the proof of Lemma A2. It suffices to consider log-spm of $h$. Note that Lemma A2 implies that $v_{H}$ is absolutely continuous with respect to $v_{L}$ and that supp $\left[K\left(\cdot ; \theta_{H}\right)\right] \geq \operatorname{supp}\left[K\left(\cdot ; \theta_{L}\right)\right]$ in the strong set order.

First, notice that if $a \geq b$, then $h$ is log-spm in $(s ; \theta)$ a.e. $-\mu$. To see this, observe that $a \geq b$ implies that $h\left(s, \theta_{H}\right)=0$ and $h\left(s, \theta_{L}\right)=$ 2 for $s$ on $\operatorname{supp}\left[K\left(\cdot ; \theta_{L}\right)\right]$, while $h\left(s, \theta_{L}\right)=0$ and $h\left(s, \theta_{H}\right)=2$ on supp $\left[K\left(\cdot ; \theta_{H}\right)\right]$. This implies that $h(s ; \theta)$ is log-spm.

Now, suppose that $a<b$. Let $B=\operatorname{supp}\left[K\left(\cdot ; \theta_{L}\right)\right] \cap \operatorname{supp}$ $\left[K\left(\cdot ; \theta_{H}\right)\right]$, which we have shown is equivalent to $D \cap[a, b]$. Pick any $s_{L}, s_{H} \in B$, and define $S_{L}(\epsilon)=\left(s_{L}-\epsilon, s_{L}\right]$ and $S_{H}(\epsilon)=\left(s_{H}-\right.$ $\left.\epsilon, s_{H}\right]$, such that $s_{H}-\epsilon \geq s_{L}$. For the moment, we will suppress the $\epsilon$ in our notation. Suppose further that $s_{L}, s_{H} \in B$, but $v_{H}\left(S_{H}\right) \cdot$ $v_{L}\left(S_{L}\right)<v_{H}\left(S_{L}\right) \cdot v_{L}\left(S_{H}\right)$. By definition, if $s_{L}>a$, then $v_{H}\left(\left[a, s_{L}-\right.\right.$ $\epsilon])>0$; then, by absolute continuity, $v_{L}\left(\left[a, s_{L}-\epsilon\right]\right)>0$, and thus $K\left(s_{L}-\epsilon ; \theta_{L}\right)>0$. Let $g(s ; \delta)$ be defined as follows:

$$
\begin{aligned}
g(s ; \delta)= & \begin{cases}-\delta \cdot \frac{v_{L}\left(\left[s_{L}, \infty\right)\right)-v_{L}\left(S_{H}\right)}{K\left(s_{L}-\epsilon ; \theta_{L}\right)} & s \in\left(-\infty, s_{L}-\epsilon\right) \\
-1 & s \in S_{L}\end{cases} \\
& \begin{cases}\delta & s \in\left[s_{L}, \infty\right) S_{H} \\
\frac{v_{L}\left(S_{L}\right)}{v_{L}\left(S_{H}\right)} & s \in S_{H} .\end{cases}
\end{aligned}
$$

It is straightforward to verify that there exists a $\delta>0$ such that the single-crossing property fails with this $g$. But this implies that for any $\epsilon$ positive and in the relevant range, $v_{H}\left(S_{H}(\epsilon)\right) \cdot$ $v_{L}\left(S_{L}(\epsilon)\right) \geq v_{H}\left(S_{L}(\epsilon)\right) \cdot v_{L}\left(S_{H}(\epsilon)\right)$. But this implies that $h$ is log-spm in $(s, \theta)$ a.e. $C$ on $B$. Since $\operatorname{supp}\left[K\left(\cdot ; \theta_{H}\right)\right] \geq \operatorname{supp}\left[K\left(\cdot ; \theta_{L}\right)\right]$, this implies that $h$ is $\log$-spm in $(s, \theta)$ a.e.- $C$ on $D$.

Extensions to Lemma 5. Part (i). Case (a) follows from Lemma A1. Case (b): Consider the case where $g$ crosses 0 (otherwise, the expectation is always nonnegative) at $s=s_{0}$, and $k>0$; 
the other cases can be handled in a manner similar to the proof of Lemma A1. Then, we extend (2) as follows:

$$
\begin{aligned}
\int g\left(s ; \theta_{H}\right) k\left(s ; \theta_{H}\right) d \mu(s) \geq & \lim _{s \rightarrow s_{0}} \frac{g\left(s ; \theta_{H}\right) k\left(s ; \theta_{H}\right)}{g\left(s ; \theta_{L}\right) k\left(s ; \theta_{L}\right)} \\
& \times\left[-\int_{-\infty}^{s_{0}}\left|g\left(s ; \theta_{L}\right)\right| k\left(s ; \theta_{L}\right) d \mu(s)\right. \\
& \left.+\int_{s_{0}}^{\infty} g\left(s ; \theta_{L}\right) k\left(s ; \theta_{L}\right) d \mu(s)\right] \\
= & \lim _{s \rightarrow s_{0}} \frac{g\left(s ; \theta_{H}\right) k\left(s ; \theta_{H}\right)}{g\left(s ; \theta_{L}\right) k\left(s ; \theta_{L}\right)} \int g\left(s ; \theta_{L}\right) k\left(s ; \theta_{L}\right) d \mu(s) .
\end{aligned}
$$

The inequality follows, as in Theorem 2 , because $g$ crosses zero at $s_{0}$ and $\left[g\left(s ; \theta_{H}\right) k\left(s ; \theta_{H}\right)\right] /\left[g\left(s ; \theta_{L}\right) k\left(s ; \theta_{L}\right)\right]$ is nonnegative and nondecreasing. Part (ii): Sufficiency follows from Lemma A2. Necessity of (A) follows from Lemma 5. Necessity of (B) follows by Lemma A2, since once the properties of Lemma A2 are established, the proof of Lemma 5 applies. Part (iii) follows from Lemma A1.

Proof of Proposition 3. (A) and (B) imply (C): if $\pi$ is differentiable in $x$ and $B$ is a convex set, we can analyze whether $\int u^{\prime}(\pi(x, s)) \pi_{x}(x, s) f(s ; \theta) d \mu(s)$ satisfies SC1. We can let $g=\pi_{x}$, and let $k=u_{1} f$, and apply Theorem 2 . Now consider the investor's choice between two values of $x, x_{H}>x_{L}$. Define $g(s, \theta) \equiv$ $u\left(\pi\left(x_{H}, s\right), \theta\right)-u\left(\pi\left(x_{L}, s\right), \theta\right)$, and let $k(s ; \theta) \equiv f(s ; \theta)$. First, observe that SC2 is preserved under monotone transformations, so that if $u$ is nondecreasing in its first argument, then by (A), $u(\pi(x, s), \theta)$ must satisfy SC2 in $(x ; s)$, and $g(s, \theta)$ satisfies SC1 in $s$. Let $s_{0}$ be the crossing point of $g(\cdot, \theta)$. First restrict attention to $s \geq s_{0}$, where $\pi\left(x_{H}, s\right) \geq \pi\left(x_{L}, s\right)$. Define $h(a, b, \theta) \equiv \int_{y=a}^{b} u_{1}(y, \theta) d y$, and note that $h$ is log-spm in $(a, b, \theta)$ for all $a<b$, by Lemmas 2 and 3 and (B). This in turn implies that $g(s, \theta)=h\left(\pi\left(x_{L}, s\right), \pi\left(x_{H}, s\right), \theta\right)$ is log-spm in $(s, \theta)$ on $s \geq s_{0}$ since $\pi$ is nondecreasing in $s$, and thus $g\left(s ; \theta_{H}\right)$ / $g\left(s ; \theta_{L}\right)$ is nondecreasing in $s$ on $s \geq s_{0}$. On the other hand, if $s<$ $s_{0}, \pi\left(x_{H}, s\right) \leq \pi\left(x_{L}, s\right)$, and $g(s, \theta)=-h\left(\pi\left(x_{L}, s\right), \pi\left(x_{H}, s\right), \theta\right)$. Then, $g\left(s ; \theta_{H}\right) / g\left(s ; \theta_{L}\right)$ is nondecreasing in $s$ on $s<s_{0}$ since $h$ is log-spm, and we apply extension (i) of Lemma 5. 
Necessity follows by Theorem 2 for the case where $\pi$ is differentiable; the proof is omitted for the more general case.

Proof of Lemma 7. Consider sufficiency first. The argument is easiest to see when $g$ is absolutely continuous with $\lim g(\mathrm{~s})=$ $\bar{g}$, so that integration by parts may be used. We also work backwards in establishing the single-crossing property: we show that $G\left(\theta_{H}\right) \leq(<) 0$ implies that $G\left(\theta_{L}\right) \leq(<) 0$. To proceed, observe that $G\left(\theta_{H}\right) \leq 0$ if and only if

$$
\bar{g}-\int g^{\prime}(s) K\left(s ; \theta_{H}\right) d s \leq 0,
$$

which requires $-\int g^{\prime}(s) K\left(s ; \theta_{H}\right) d s \leq 0$ since $g$ is single crossing. But, quasi concavity of $g$ implies that $-g^{\prime}$ is SC1. Thus, equation (2) can be applied, letting $-g^{\prime}$ play the role of $g$, $K$ play the role of $k$, so that, if we define $L(s) \equiv K\left(s ; \theta_{H}\right) / K\left(s ; \theta_{L}\right)$, we have

$$
-\int g^{\prime}(s) K\left(s ; \theta_{H}\right) d s \geq-L\left(s_{0}\right) \int g^{\prime}(s) K\left(s ; \theta_{L}\right) d s .
$$

Now, since $K$ is a probability distribution, if $L(\cdot)$ is nondecreasing, it must always be less than one. Thus, if $-\int g^{\prime}(s) K\left(s ; \theta_{H}\right) d s \leq 0$, then $-\int g^{\prime}(s) K\left(s ; \theta_{H}\right) d s \geq-\int g^{\prime}(s) K\left(s ; \theta_{L}\right) d s$. But then,

$$
\begin{aligned}
G\left(\theta_{L}\right) & =\bar{g}-\int g^{\prime}(s) K\left(s ; \theta_{L}\right) d s \\
& \leq \bar{g}-\int g^{\prime}(s) K\left(s ; \theta_{H}\right) d s \\
& =G\left(\theta_{H}\right),
\end{aligned}
$$

and we have established that $G\left(\theta_{H}\right) \leq(<) 0$ implies $G\left(\theta_{L}\right) \leq(<)$ 0 . Now consider necessity. Suppose that $K$ is not log-supermodular. Then, there exists an open interval $\left(s_{0}, s_{0}+\epsilon\right)$ such that $\left(K\left(s_{0}+\epsilon ; \theta\right)-K\left(s_{0} ; \theta\right)\right) / K\left(s_{0} ; \theta\right)$ is decreasing in $\theta$. Then, let $g(s ; \delta)$ be defined as follows:

$$
g(s ; \delta)=\left\{\begin{array} { c l } 
{ - 1 } & { s \in ( - \infty , s _ { 0 } ] } \\
{ \frac { K ( s _ { 0 } ; \theta _ { L } ) - \delta ( 1 - K ( s _ { 0 } + \epsilon ; \theta _ { L } ) ) } { K ( s _ { 0 } + \epsilon ; \theta _ { L } ) - K ( s _ { 0 } ; \theta _ { L } ) } } & { s \in ( s _ { 0 } , s _ { 0 } + \epsilon ] }
\end{array} \left\{\begin{array}{ll}
\delta \quad s \in\left(s_{0}+\epsilon, \infty\right)
\end{array}\right.\right.
$$

so that $G\left(\theta_{L}\right)=0$, while for $\delta$ sufficiently small, $G\left(\theta_{H}\right)<0$. 
Proof of Lemma 8. (i): Under the assumptions of the theorem, $v(x, y, s)$ satisfies $(\mathrm{SM})$ if and only if $u(x, s ; b) \equiv v(x, b(x), s)$ has SC2 in $(x ; s)$ for all functions $b$. Furthermore, $V(x, y, \theta)$ satisfies $(\mathrm{SM})$ if and only if $U(x, \theta ; b) \equiv V(x, b(x), \theta)$ has SC2 in $(x ; \theta)$ for all functions $b$. So, if we know that $v(x, y, s)$ satisfies (SM), then $u(x, s ; b)$ has SC2 in $(x ; \mathrm{s})$ for all functions $b$. If $k$ is log-spm a.e. $\mu$, then Theorem 2 implies that $U(x, \theta ; \mathbf{b})$ has SC2 in $(x ; s)$ for all functions $b$. But this in turn implies that $V(x, y, \theta)$ satisfies (SM).

(ii): Consider any $f: \mathrm{S} \times \Theta \rightarrow \mathbb{R}_{+}$. Let $F(s ; \theta)=\int_{-\infty}^{s} f(t ; \theta) d \mu(t)$. The working paper [Athey 1996] shows that if $F(\cdot ; \theta)$ is not ordered by (MLR), then there exists a continuous $g$ which satisfies $\mathrm{SC} 1$ so that $\int g(s) d F(s ; \theta)$ fails $\mathrm{SC} 1$ (this is a continuous approximation to the test functions from Lemma 6). Consider this function $g$. We know that, since $g$ is continuous and crosses zero only once, it must be monotone nondecreasing in some neighborhood of the crossing point/region. Now, define the following points, which are the boundaries of the region, where $g(s)=0: c=\inf _{s \in \mathbb{R}}\{s$ : $g(s)=0\}, d=\sup _{s \in \mathbb{R}}\{s: g(s)=0\}$. Now, we can find a $\delta>0$ and two corresponding points, $c_{\delta} \equiv \sup _{s<c}\{s \mid g(s)=-\delta\}$ and $d_{\delta} \equiv \inf _{s>d}$ $\{s \mid g(s)=\delta\}$, such that $(c, d) \subset\left(c_{\delta}, d_{\delta}\right)$ and $g$ is nondecreasing on $\left(c_{\delta}, d_{\delta}\right)$. Let us define a new function, $\alpha(s)$, as follows: $\alpha(s)=\delta$ for $s \in\left(c_{\delta}, d_{\delta}\right), \alpha(s)=|g(s)|$ elsewhere. Now, pick any $x_{H}>x_{L}$, and let $v(x, y, s)=x \cdot g(s) /\left(x_{H}-x_{L}\right)+\alpha(s) \cdot y$. Since $\alpha(s)$ and $g(s)$ are continuous and $\alpha(s)>0, v$ satisfies (WB). Finally,

$$
\frac{\partial v}{\partial x} / \frac{\partial v}{\partial y}=g(s) /\left(\left(x_{H}-x_{L}\right) \cdot \alpha(s)\right)
$$

is nondecreasing in $s$. Thus, $v$ satisfies the assumptions of the theorem as well as (SM). Now, $\int v(x, y, s) d F(s ; \theta)$ satisfies (SM) if and only if $\int v(x, b(x), s) d F(s ; \theta)$ satisfies SC2 in $(x ; \theta)$ for all $b$. Let $b(x)=0$. But, $v\left(x_{H}, 0, s\right)-v\left(x_{L}, 0, s\right)=g(s)$, and by construction $\int g(s) d F(s ; \theta)$ fails $\mathrm{SC} 1$, which in turn implies that $\int v(x, 0, s)$ $d F(s ; \theta)$ fails SC2 in $(x ; \theta)$. Thus, $\int v(x, y, s) d F(s ; \theta)$ fails (SM).

(iii): If $v(x, y, s)$ fails the (SM), then there exists a $b(x)$ such that $v(x, b(x), s)$ fails SC2 in $(x ; s)$. But then, Theorem 2 implies that there exists an $f(s ; \theta)$ which is log-spm a.e. $\mu$ such that $\int v(x, b(x), s) f(s ; \theta) d \mu(s)$ fails SC2 in $(x ; \theta)$. We conclude that $\int v(x, y, s) f(s ; \theta) d \mu(s)$ fails (SM). 


\section{REFERENCES}

Ahlswede, Rudolf, and David Daykin, "An Inequality for Weights of Two Families of Sets, their Unions and Intersections," Zeitschrift für Wahrscheinlichkeitstheorie und Verwandte Gebiete, XCIII (1979), 183-185.

Athey, Susan, "Comparative Statics under Uncertainty: Single Crossing Properties and Log-Supermodularity," MIT Working Paper No. 96-22, 1996.

, "Characterizing Properties of Stochastic Objective Functions," MIT Working Paper No. 96-1R, 1998.

"Investment and Information in a Risk-Averse Firm," MIT Working Paper 00-30, 2000.

"Single Crossing Properties and the Existence of Pure Strategy Nash Equilibria in Games of Incomplete Information," Econometrica, LXIX (2001), $861-890$.

Diamond, Peter, and Joseph Stiglitz, "Increases in Risk and Risk Aversion," Journal of Economic Theory, VIII (1974), 337-360.

Eeckhoudt, Louis, and Christian Gollier, "Demand for Risky Assets and the Monotone Probability Ratio Order," Journal of Risk and Uncertainty, XI (1995), 113-122.

Gollier, Christian, "The Comparative Statics of Changes in Risk Revisited," Journal of Economic Theory, LXVI (1995), 522-535.

Gollier, Christian, and Miles Kimball, "Toward a Systematic Approach to the Economic Effects of Uncertainty I: Comparing Risks," mimeo, IDEI, Toulouse, France, 1995a.

Gollier, Christian, and Miles Kimball, "Toward a Systematic Approach to the Economic Effects of Uncertainty II: Characterizing Utility Functions," mimeo, IDEI, Toulouse, France, 1995b.

Hadar, Josef, and William Russell, "Applications in Economic Theory and Analysis," in Stochastic Dominance, G. Whitmore and M. Findlay, eds. (Lexington, MA: Lexington Books, 1978).

Jewitt, Ian, "A Note on Comparative Statics and Stochastic Dominance," Journal of Mathematical Economics, XV (1986), 249-254.

_- "Risk Aversion and the Choice between Risky Prospects: The Preservation of Comparative Statics Results," Review of Economic Studies, LIV (1987), $73-85$.

_- "Justifying the First Order Approach to Principal-Agent Problems," Econometrica, LVI (1988a), 1177-1190.

, "Risk and Risk Aversion in the Two Risky Asset Portfolio Problem," mimeo, University of Bristol, Bristol, UK, 1988b.

_ - "Choosing between Risky Prospects: The Characterization of Comparative Statics Results, and Location Independent Risk," Management Science, XXXV (1989), 60-70.

Karlin, Samuel, Total Positivity: Volume I (Stanford, CA: Stanford University Press, 1968).

Karlin, Samuel, and Yosef Rinott, "Classes of Orderings of Measures and Related Correlation Inequalities. I. Multivariate Totally Positive Distributions," Journal of Multivariate Analysis, X (1980), 467-498.

Karlin, Samuel, and Herman Rubin, "The Theory of Decision Procedures for Distributions with Monotone Likelihood Ratio," Annals of Mathematical Statistics, XXVII (1956), 272-299.

Kimball, Miles, "Precautionary Savings in the Small and in the Large," Econometrica, LVIII (1990), 53-73.

_. "Standard Risk Aversion," Econometrica, LXI (1993), 589-611.

Landsberger, Michael, and Isaac Meilijson, "Demand for Risky Financial Assets: A Portfolio Analysis," Journal of Economic Theory, L (1990), 204-213.

Lehmann, Eric, "Ordered Families of Distributions," Annals of Mathematical Statistics, XXVI (1955), 399-419.

Lorentz, George, "An Inequality for Rearrangement," American Mathematical Monthly, LX (1953), 176-179.

Meyer, Jack, and Michael Ormiston, "The Comparative Statics of Cumulative Distribution Function Changes for the Class of Risk Averse Agents," Journal of Economic Theory, XXXI (1983), 153-169. 
Meyer, Jack, and Michael Ormiston, "Strong Increases in Risk and Their Comparative Statics," International Economic Review, XXVI (1985), 425-437.

Meyer, Jack, and Michael Ormiston, "Deterministic Transformations of Random Variables and the Comparative Statics of Risk," Journal of Risk and Uncertainty, II (1989), 179-188.

Milgrom, Paul, "Good News and Bad News: Representation Theorems and Applications," Bell Journal of Economics, XII (1981), 380-391.

_- "Comparing Optima: Do Simplifying Assumptions Affect Conclusions?" Journal of Political Economy, CII (1994), 607-615.

Milgrom, Paul, and John Roberts, "The Economics of Modern Manufacturing: Technology, Strategy, and Organization," American Economic Review, LXXX (1990a), 511-528.

Milgrom, Paul, and John Roberts, "Rationalizability, Learning, and Equilibrium in Games with Strategic Complementarities," Econometrica, LVIII (1990b), $1255-1277$.

Milgrom, Paul, and John Roberts, "Comparing Equilibria," American Economic Review, LXXXIV (1994), 441-459.

Milgrom, Paul, and Christina Shannon, "Monotone Comparative Statics," Econometrica, LXII (1994), 157-180.

Milgrom, Paul, and Robert Weber, "A Theory of Auctions and Competitive Bidding," Econometrica, L (1982), 1089-1122.

Ormiston, Michael, "First and Second Degree Transformations and Comparative Statics under Uncertainty," International Economic Review, XXXIII (1992), $33-44$.

Ormiston, Michael, and Edward Schlee, "Necessary Conditions for Comparative Statics under Uncertainty," Economics Letters, XL (1992), 429-434.

Ormiston, Michael, and Edward Schlee, "Comparative Statics under Uncertainty for a Class of Economic Agents," Journal of Economic Theory, LXI (1993), $412-422$.

Pratt, John, "Aversion to One Risk in the Presence of Others," Journal of Risk and Uncertainty, I (1988), 395-413.

Sandmo, Agmar, "On the Theory of the Competitive Firm under Price Uncertainty," American Economic Review, LXI (1971), 65-73.

Scarsini, Marco, "Comparing Risk and Risk Aversion," in Stochastic Orders and Their Applications, Moshe Shaked and George Shanthikumar, eds. (New York, NY: Academic University Press, 1994).

Spulber, Daniel, "Bertrand Competition When Rivals' Costs Are Unknown," Journal of Industrial Economics, XLIII (1995), 1-11.

Topkis, Donald, "Minimizing a Submodular Function on a Lattice," Operations Research, XXVI (1978), 305-321.

_. "Equilibrium Points in Nonzero-Sum n-person Submodular Games," Siam Journal of Control and Optimization, XVII (1979), 773-787.

Vives, Xavier, "Nash Equilibrium with Strategic Complementarities," Journal of Mathematical Economics, XIX (1990), 305-321.

Whitt, Ward, "Multivariate Monotone Likelihood Ratio Order and Uniform Conditional Stochastic Order," Journal of Applied Probability, XIX (1982), 695-701. 


\section{This article has been cited by:}

1. Jianqing Chen, De Liu, Andrew B Whinston. 2009. Auctioning Keywords in Online Search. Journal of Marketing 73:4, 125-141. [CrossRef]

2. Bruno H. Strulovici, Thomas A. Weber. 2009. Generalized monotonicity analysis. Economic Theory . [CrossRef]

3. Scott Ashworth, Ethan Bueno de Mesquita. 2008. Electoral Selection, Strategic Challenger Entry, and the Incumbency Advantage. The Journal of Politics 70:04, 1006. [CrossRef]

4. Ignacio Esponda. 2008. Behavioral Equilibrium in Economies with Adverse Selection. American Economic Review 98:4, 1269-1291. [CrossRef]

5. Arthur Hau. 2008. When Is a Coinsurance-Type Insurance Policy Inferior or Even Giffen?. Journal of Risk \& Insurance 75:2, 343-364. [CrossRef]

6. B. H. Strulovici, T. A. Weber. 2008. Monotone Comparative Statics: Geometric Approach. Journal of Optimization Theory and Applications 137:3, 641-673. [CrossRef]

7. Robert G. Chambers, J. Quiggin. 2008. Comparative statics for state-contingent technologies. Journal of Economics 93:2, 203-214. [CrossRef]

8. Christian Gollier. 2007. Whom should we believe? Aggregation of heterogeneous beliefs. Journal of Risk and Uncertainty 35:2, 107-127. [CrossRef]

9. David W. Archer, Thomas D. Crocker, Jason F. Shogren. 2006. Choosing Children's Environmental Risk. Environmental \& Resource Economics 33:3, 347-369. [CrossRef]

10. LEONID KOGAN, STEPHEN A. ROSS, JIANG WANG, MARK $M$. WESTERFIELD. 2006. The Price Impact and Survival of Irrational Traders. The Journal of Finance 61:1, 195-229. [CrossRef]

11. Scott Ashworth, Ethan Bueno de Mesquita. 2006. Monotone Comparative Statics for Models of Politics. American Journal of Political Science 50:1, 214-231. [CrossRef]

12. Jorge Ibarra-Salazar. 2005. The Newsboy Model: Changes in Risk and Price. The Geneva Risk and Insurance Review 30:1, 99-109. [CrossRef]

13. Alexander E. Saak. 2003. Spatial and Temporal Marketing Considerations under Marketing Loan Programs. American Journal of Agricultural Economics 85:4, 872-887. [CrossRef]

14. David McAdams. 2003. Isotone Equilibrium in Games of Incomplete Information. Econometrica 71:4, 1191-1214. [CrossRef]

15. Mathias Dewatripont, Patrick Legros, Steven A. Matthews . 2003. Moral Hazard and Capital Structure DynamicsMoral Hazard and Capital Structure Dynamics. Journal of the European Economic Association 1:4, 890-930. [Abstract] [PDF] [PDF Plus] 
16. Jutta Roosen, David A. Hennessy. 2003. Tests for the Role of Risk Aversion on Input Use. American Journal of Agricultural Economics 85:1, 30-43. [CrossRef]

17. Nicolaj Siggelkow. 2002. Misperceiving Interactions Among Complements and Substitutes: Organizational Consequences. Management Science 48:7, 900-916. [CrossRef] 